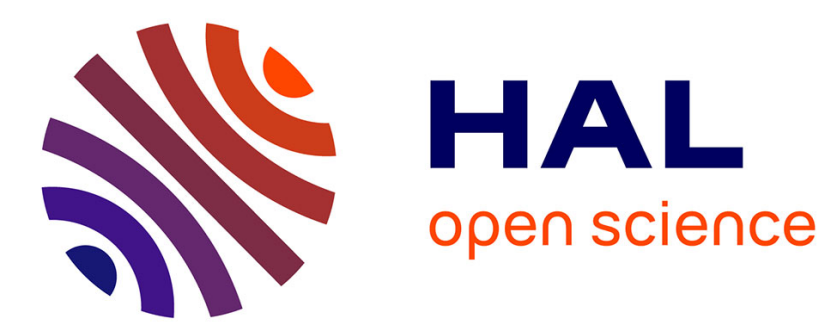

\title{
Topological sensitivity of energy cost functional for wave-based defect identication
}

Marc Bonnet

\section{To cite this version:}

Marc Bonnet. Topological sensitivity of energy cost functional for wave-based defect identication. Comptes Rendus Mécanique, 2010, 338, pp.377-389. 10.1016/j.crme.2010.07.016 • hal-00505494

\section{HAL Id: hal-00505494 \\ https://hal.science/hal-00505494}

Submitted on 19 Aug 2010

HAL is a multi-disciplinary open access archive for the deposit and dissemination of scientific research documents, whether they are published or not. The documents may come from teaching and research institutions in France or abroad, or from public or private research centers.
L'archive ouverte pluridisciplinaire HAL, est destinée au dépôt et à la diffusion de documents scientifiques de niveau recherche, publiés ou non, émanant des établissements d'enseignement et de recherche français ou étrangers, des laboratoires publics ou privés. 


\title{
Topological sensitivity of energy cost functional for wave-based defect identification
}

\author{
Marc Bonnet ${ }^{\mathrm{a}}$, \\ ${ }^{a}$ Laboratoire de mécanique des Solides (UMR CNRS 7649), École Polytechnique, F-91128 Palaiseau cedex, France \\ Received $* * * * * ;$ accepted after revision +++++ \\ Presented by $\mathrm{xxxxx}$
}

\begin{abstract}
This article is concerned with establishing the topological sensitivity (TS) against the nucleation of small trial inclusions of an energy-like cost function. The latter measures the discrepancy between two time-harmonic elastodynamic states (respectively defined, for cases where overdetermined boundary data is available for identification purposes, in terms of Dirichlet or Neumann boundary data for the same reference solid) as the strain energy of their difference. Such cost function constitutes a particular form of error in constitutive relation and may be used for e.g. defect identification. The TS is expressed in terms of four elastodynamic fields, namely the free and adjoint solutions for Dirichlet or Neumann data. A similar result is also given for the linear acoustic scalar case. A synthetic numerical example where the TS result is used for the qualitative identification of an inclusion is presented for a simple 2D acoustic configuration. To cite this article: M. Bonnet, C. R. Mécanique 338 (2010).
\end{abstract}

Résumé

Gradient topologique d'une fonction-coût énergétique pour l'identification de défauts en élastodynamique. Cet article porte sur la formulation du champ de gradient topologique (GT), correspondant à l'apparition de petites inclusions virtuelles dans le milieu, d'une fonction-coût de type énergétique. Cette dernière quantifie l'écart entre deux états élastodynamiques en régime fréquentiel (respectivement définis en termes de données de type Dirichlet ou Neumann sur la frontière pour des situations d'identification exploitant la possession de données aux limites surabondantes) comme l'énergie de déformation de leur différence. Ce type de fonctioncoût constitue une forme particulière d'erreur en relation de comportement et peut être utilisée par exemple pour l'identification de défauts. Le GT est exprimée en termes de quatre champs élastodynamiques, à savoir les champs libre et adjoint associés à chaque type de données aux limites. Un résultat similaire est également donné pour le cas de l'acoustique linéaire. L'application du résultat à l'identification qualittative d'une inclusion est présenté sur une configuration 2D acoustique simple. Pour citer cet article : M. Bonnet, C. R. Mécanique 338 (2010).

Key words: Topological sensitivity; Energy-like functional; Elastodynamics; Defect identification

Mots-clés : Gradient topologique ; Fonction-coût énergétique ; Elastodynamique ; Identification de défauts

Email address: bonnet@lms.polytechnique.fr (Marc Bonnet).

Preprint submitted to Elsevier Science

21 July 2010 


\section{Preliminaries}

\subsection{Introduction}

Defects in solids can be viewed as local perturbations of the properties of the reference, i.e. defect-free, solid. Identification of such defects from overdetermined data is often formulated as the minimization of a cost functional expressing the misfit between actual data and its simulated counterpart for a trial configuration of the defect(s). Energy-like cost functionals are especially relevant for this kind of problem as they evaluate the mismatch as the energy of the difference between statically (or dynamically) admissible stresses and and stresses corresponding to kinematically admissible displacements through a given (possibly spatially varying) constitutive model. Such energy-like functionals have been introduced for the identification of scalar spatially-varying conductivity coefficients in e.g. [19-21], with mathematical and numerical issues also discussed in e.g. $[10,15]$. In solid mechanics, such functionals, often referred to as error in constitutive relation (ECR) functionals, have been initially introduced for error estimation in FEM computations [23] before being also applied to various identification problems [11, 13, 14, 22]. Energy-like functionals are also found to be useful for solving data completion (Cauchy) problems [4].

Here, an energy-like cost functional $E$ is considered for inclusion identification problems using 3D elastodynamic data. Iterative minimization algorithms applied to $E$ (and other cost functionals as well) entail high computational costs as each evaluation of $E$ requires one full forward solution. Alternative approaches for solving inverse scattering problems have appeared, in particular "sampling" methods based on the definition of a defect indicator function (see e.g. [8,28] and references therein) and methods based on small-defect asymptotics [1,9]. Identification approaches based on the topological sensitivity (TS) combine sampling and small-defect asymptotics, as they quantify the perturbation undergone by the cost functional due to the hypothetical nucleation of a small defect of prescribed nature and shape at any given sampling location. TS-based formulations for cost functionals defined in terms of boundary integrals, typically associated to boundary measurements, are presented in e.g. [7, 12, 16, 25], and their usefulness for qualitative identification demonstrated.

In this article, the TS of an energy-like cost functional $E$ is established. This functional is expressed in terms of domain integrals whose support contains the trial inclusion and which involve displacement gradients, as it evaluate the strain energy of the discrepancy between Neumann and Dirichlet solutions. Finding the TS of $E$ therefore entails an analysis substantially different from that, considered in most previous works on this topic, yielding the TS of functionals whose support does not intersect the trial defect. While the TS of an energy functional is established in [5] for the identification of bubbles in Stokes flows, such treatment has not, to our best knowledge, been proposed so far for elastodynamics. In an effort to bridge this gap, the main goal of this article is to establish the TS of an energy-like functional for 3D time-harmonic elastodynamics. Although one specific form of functional is considered here, the method is expected to carry over to other known forms of ECR functionals. The main derivation is presented in Section 2, leading to a TS formulation in terms of two adjoint solutions. Essentially the same treatment can be applied to scalar linear acoustic problems, which is done in abbreviated fashion in Section 3. A simple numerical example for the 2D Helmholtz equation is presented in Section 4.

\subsection{Inverse problem.}

Consider an inverse scattering problem where the reference homogeneous solid $\Omega$ with boundary $S$, containing a bonded inclusion $B_{\text {true }}$ with boundary $\Gamma_{\text {true }}$ (or a set thereof), is interrogated by elastic waves. The reference medium is characterized by its elastic tensor $\mathcal{C}$ and mass density $\rho$; the respective material characteristics of the inclusion are denoted as $\mathcal{C}_{\text {true }}^{\star}$ and $\rho_{\text {true }}^{\star}$. 
To identify the hidden defect $B_{\text {true }}$, time-harmonic excitations are applied in the form of volume $(\boldsymbol{f})$ and surface $(\boldsymbol{g})$ force densities having respective supports $V \subset \Omega$ and $S_{\mathrm{T}} \subset S$, and displacements $\boldsymbol{U}$ over the complementary external surface $S_{u}=S \backslash S_{\mathrm{T}}$. The implicit time-harmonic factor $\exp (\mathrm{i} \omega t)$ where $\omega$ denotes the angular frequency of excitation is, as usual, omitted hereon. Letting $B$ denote a trial inclusion bounded by $\Gamma$, the prescribed excitation $(\boldsymbol{f}, \boldsymbol{g}, \boldsymbol{U})$ gives rise to elastodynamic displacement fields $\boldsymbol{u}_{B}=\boldsymbol{u}_{B}\left[B, \mathcal{C}^{\star}, \rho^{\star}\right]$ in $B \cup(\Omega \backslash \bar{B})$.

For identification purposes, the displacement $\boldsymbol{u}_{\text {obs }}$ induced in the flawed solid by $(\boldsymbol{f}, \boldsymbol{g}, \boldsymbol{U})$ is monitored over the measurement surface $S_{\mathrm{obs}} \subset S_{\mathrm{T}}$ (other possibilities, e.g. finite sets of measurement points, being also allowed by the ensuing treatment). Under perfect modelling and experimental conditions, one has $\boldsymbol{u}_{B}\left[B_{\text {true }}, \mathcal{C}_{\text {true }}^{\star}, \rho_{\text {true }}^{\star}\right]=\boldsymbol{u}_{\text {obs }}$ on $S_{\text {obs }}$. In this article, the identification problem is formulated as a minimisation problem for a cost function which evaluates the strain energy of the difference between 'Neumann' and 'Dirichlet' solutions differing (for a given trial inclusion $B$ ) only by the boundary data on $S_{\text {obs }}$ (taken as either the given load $\left.\boldsymbol{g}\right|_{S_{\mathrm{obs}}}$ or the measured displacement $\boldsymbol{u}_{\mathrm{obs}}$ ). Accordingly, the 'Neumann' and 'Dirichlet' free fields $\boldsymbol{u}^{\mathrm{N}}$ and $\boldsymbol{u}^{\mathrm{D}}$ arising in the reference solid $\Omega$ are defined by the weak formulations

$$
\begin{array}{lll}
\text { find } \boldsymbol{u}^{\mathrm{N}} \in \mathcal{V}_{\mathrm{N}}, \mathcal{Z}\left(\boldsymbol{u}^{\mathrm{N}}, \boldsymbol{w}\right)=\mathcal{F}(\boldsymbol{w})+\mathcal{F}_{\mathrm{N}}(\boldsymbol{w}) & \left(\forall \boldsymbol{w} \in \mathcal{V}_{\mathrm{N}, 0}\right) \\
\text { find } \boldsymbol{u}^{\mathrm{D}} \in \mathcal{V}_{\mathrm{D}}, \mathcal{Z}\left(\boldsymbol{u}^{\mathrm{D}}, \boldsymbol{w}\right)=\mathcal{F}(\boldsymbol{w}) & & \left(\forall \boldsymbol{w} \in \mathcal{V}_{\mathrm{D}, 0}\right)
\end{array}
$$

In $(1 \mathrm{a}, \mathrm{b})$ the dynamic stiffness bilinear form $\mathcal{Z}$ for the reference solid is defined by

$$
\mathcal{Z}(\boldsymbol{u}, \boldsymbol{w})=\int_{\Omega} \boldsymbol{\nabla} \boldsymbol{u}: \mathcal{C}: \nabla \boldsymbol{w} \mathrm{d} V-\omega^{2} \int_{\Omega} \rho \boldsymbol{u} \cdot \boldsymbol{w} \mathrm{d} V=\mathcal{K}(\boldsymbol{u}, \boldsymbol{w})-\omega^{2} \mathcal{M}(\boldsymbol{u}, \boldsymbol{w}) .
$$

in terms of the reference stiffness and mass operators $\mathcal{K}, \mathcal{M}$; the linear forms $\boldsymbol{w} \mapsto \mathcal{F}(\boldsymbol{w})$ and $\boldsymbol{w} \mapsto \mathcal{F}_{\mathrm{N}}(\boldsymbol{w})$ correspond to the loads applied outside of $S_{\text {obs }}$ and over $S_{\text {obs }}$, respectively and are defined by

$$
\mathcal{F}(\boldsymbol{w})=\int_{V} \boldsymbol{f} \cdot \boldsymbol{w} \mathrm{d} V+\int_{S_{\mathrm{T}} \backslash S_{\mathrm{obs}}} \boldsymbol{g} \cdot \boldsymbol{w} \mathrm{d} S, \quad \mathcal{F}_{\mathrm{N}}(\boldsymbol{w})=\int_{S_{\mathrm{obs}}} \boldsymbol{g} \cdot \boldsymbol{w} \mathrm{d} S
$$

and the various spaces of admissible displacements $\mathcal{V}_{\mathrm{N}}, \mathcal{V}_{\mathrm{N}, 0}, \mathcal{V}_{\mathrm{D}}, \mathcal{V}_{\mathrm{D}, 0}$ are defined by

$$
\begin{aligned}
\mathcal{V}_{\mathrm{N}} & =\left\{\boldsymbol{w} \in H^{1}(\Omega), \boldsymbol{w}=\boldsymbol{U} \text { on } S_{u}\right\} \\
\mathcal{V}_{\mathrm{N}, 0} & =\left\{\boldsymbol{w} \in H^{1}(\Omega), \boldsymbol{w}=\mathbf{0} \text { on } S_{u}\right\} \\
\mathcal{V}_{\mathrm{D}} & =\left\{\boldsymbol{w} \in H^{1}(\Omega), \boldsymbol{w}=\boldsymbol{U} \text { on } S_{u} \text { and } \boldsymbol{w}=\boldsymbol{u}_{\mathrm{obs}} \text { on } S_{\mathrm{obs}}\right\} \\
\mathcal{V}_{\mathrm{D}, 0} & =\left\{\boldsymbol{w} \in H^{1}(\Omega), \boldsymbol{w}=\mathbf{0} \text { on } S_{u} \cup S_{\mathrm{obs}}\right\}
\end{aligned}
$$

Similarly, 'Neumann' and 'Dirichlet' fields $\boldsymbol{u}_{B}^{\mathrm{N}}=\boldsymbol{u}_{B}^{\mathrm{N}}\left[B, \mathcal{C}^{\star}, \rho^{\star}\right]$ and $\boldsymbol{u}_{B}^{\mathrm{D}}=\boldsymbol{u}_{B}^{\mathrm{D}}\left[B, \mathcal{C}^{\star}, \rho^{\star}\right]$ for a trial defect are defined, for given trial inclusion $\left(B, \mathcal{C}^{\star}, \rho^{\star}\right)$ by the weak formulations

$$
\begin{array}{lll}
\text { find } \boldsymbol{u}_{B}^{\mathrm{N}} \in \mathcal{V}_{\mathrm{N}}, \mathcal{Z}_{B}\left(\boldsymbol{u}_{B}^{\mathrm{N}}, \boldsymbol{w}\right)=\mathcal{F}(\boldsymbol{w})+\mathcal{F}_{\mathrm{N}}(\boldsymbol{w}) & \left(\forall \boldsymbol{w} \in \mathcal{V}_{\mathrm{N}, 0}\right), \\
\text { find } \boldsymbol{u}_{B}^{\mathrm{D}} \in \mathcal{V}_{\mathrm{D}}, \mathcal{Z}_{B}\left(\boldsymbol{u}_{B}^{\mathrm{D}}, \boldsymbol{w}\right)=\mathcal{F}(\boldsymbol{w}) & \left(\forall \boldsymbol{w} \in \mathcal{V}_{\mathrm{D}, 0}\right),
\end{array}
$$

with the dynamic stiffness operator $\mathcal{Z}_{B}$ defined in terms of contrasts $\Delta \mathcal{C}=\mathcal{C}^{\star}-\mathcal{C}$ and $\Delta \rho=\rho^{\star}-\rho$ by

$$
\mathcal{Z}_{B}(\boldsymbol{u}, \boldsymbol{w})=\mathcal{Z}(\boldsymbol{u}, \boldsymbol{w})+\Delta \mathcal{Z}(\boldsymbol{u}, \boldsymbol{w}), \quad \Delta \mathcal{Z}(\boldsymbol{u}, \boldsymbol{w})=\int_{B} \boldsymbol{\nabla} \boldsymbol{u}: \Delta \mathcal{C}: \nabla \boldsymbol{w} \mathrm{d} V-\omega^{2} \int_{B} \Delta \rho \boldsymbol{u} \cdot \boldsymbol{w} \mathrm{d} V
$$

The energy cost functional $E\left(B, \mathcal{C}^{\star}, \rho^{\star}\right)$ considered in this article is defined (with overbars denoting complex conjugation) by

$$
E\left(B, \mathcal{C}^{\star}, \rho^{\star}\right):=\frac{1}{2} \mathcal{K}\left(\boldsymbol{u}_{B}^{\mathrm{N}}-\boldsymbol{u}_{B}^{\mathrm{D}}, \overline{\boldsymbol{u}}_{B}^{\mathrm{N}}-\overline{\boldsymbol{u}}_{B}^{\mathrm{D}}\right)
$$

As $E\left(B, \mathcal{C}^{\star}, \rho^{\star}\right) \geq 0$ by virtue of the non-negativity of $\mathcal{K}$, and $E\left(B_{\text {true }}, \mathcal{C}_{\text {true }}^{\star}, \rho_{\text {true }}^{\star}\right)=0$ for exact data $\boldsymbol{u}_{\text {obs }}$, the inclusion identification problem may be recast as the minimization of $E\left(B, \mathcal{C}^{\star}, \rho^{\star}\right)$. In this article, 
however, instead of considering the (computationally demanding) actual minimization of $E$, a preliminary identification approach based on considering the sensitivity of $E$ to the nucleation of a vanishingly small inclusion, i.e. the topological sensitivity of $E$, is developed.

\subsection{Topological sensitivity of E: definitions and notation}

Let $B_{a}(\boldsymbol{z})=\boldsymbol{z}+a \mathcal{B}$ (where $\mathcal{B} \subset \mathbb{R}^{3}$ is a fixed bounded open set of volume $|\mathcal{B}|$ containing the origin) define the region occupied by an elastic inclusion of (small) characteristic radius $a>0$, whose location $\boldsymbol{z} \in \Omega$, material properties $\mathcal{C}^{\star}, \rho^{\star}$ and shape $\mathcal{B}$ are specified (e.g. $\mathcal{B}$ is the unit ball for a spherical inclusion).

Of interest here is the leading behavior as $a \rightarrow 0$ of the energy functional (7) with $B=B_{a}(\boldsymbol{z})$. Accordingly, let $\boldsymbol{u}_{a}^{\mathrm{N}}(\cdot ; \boldsymbol{z})$ and $\boldsymbol{u}_{a}^{\mathrm{D}}(\cdot ; \boldsymbol{z})$ denote the solution to problems $(5 \mathrm{a}, \mathrm{b})$ with $B=B_{a}(\boldsymbol{z})$, and define

$$
\mathcal{E}\left(a ; \boldsymbol{z}, \mathcal{B}, \mathcal{C}^{\star}, \rho^{\star}\right)=E\left(B_{a}(\boldsymbol{z}), \mathcal{C}^{\star}, \rho^{\star}\right)=\frac{1}{2} \mathcal{K}\left(\boldsymbol{u}_{a}^{\mathrm{N}}-\boldsymbol{u}_{a}^{\mathrm{D}}, \overline{\boldsymbol{u}}_{a}^{\mathrm{N}}-\overline{\boldsymbol{u}}_{a}^{\mathrm{D}}\right)
$$

In the sequel, the dependence of $\mathcal{E}$ in $\boldsymbol{z}, \mathcal{B}, \mathcal{C}^{\star}, \rho^{\star}$ will for notational convenience be implicit, i.e. the notation $\mathcal{E}(a)$ will be used instead of $\mathcal{E}\left(a ; \boldsymbol{z}, \mathcal{B}, \mathcal{C}^{\star}, \rho^{\star}\right)$.

\section{Small-inclusion expansion of the energy cost functional}

\subsection{Formulation of expansion using adjoint solutions}

Introduce the perturbations $\boldsymbol{v}_{a}^{\mathrm{N}}=\boldsymbol{u}_{a}^{\mathrm{N}}-\boldsymbol{u}^{\mathrm{N}}$ and $\boldsymbol{v}_{a}^{\mathrm{D}}=\boldsymbol{u}_{a}^{\mathrm{D}}-\boldsymbol{u}^{\mathrm{D}}$ with respect to the free Neumann and Dirichlet fields. On subtracting $(1 \mathrm{a}, \mathrm{b})$ from $(5 \mathrm{a}, \mathrm{b})$, one readily arrives at the following governing weak formulations for $\boldsymbol{v}_{a}^{\mathrm{N}}$ and $\boldsymbol{v}_{a}^{\mathrm{D}}$ :

$$
\begin{array}{ll}
\text { find } \boldsymbol{v}_{a}^{\mathrm{N}} \in \mathcal{V}_{\mathrm{N}, 0}, \mathcal{Z}_{a}\left(\boldsymbol{v}_{a}^{\mathrm{N}}, \boldsymbol{w}\right)=-\Delta \mathcal{Z}_{a}\left(\boldsymbol{u}^{\mathrm{N}}, \boldsymbol{w}\right) & \left(\forall \boldsymbol{w} \in \mathcal{V}_{\mathrm{N}, 0}\right), \\
\text { find } \boldsymbol{v}_{a}^{\mathrm{D}} \in \mathcal{V}_{\mathrm{D}, 0}, \mathcal{Z}_{a}\left(\boldsymbol{v}_{a}^{\mathrm{D}}, \boldsymbol{w}\right)=-\Delta \mathcal{Z}_{a}\left(\boldsymbol{u}^{\mathrm{D}}, \boldsymbol{w}\right) & \left(\forall \boldsymbol{w} \in \mathcal{V}_{\mathrm{D}, 0}\right),
\end{array}
$$

By virtue of $\left(\boldsymbol{w}, \boldsymbol{w}^{\prime}\right) \mapsto \mathcal{K}\left(\boldsymbol{w}, \overline{\boldsymbol{w}}^{\prime}\right)$ being Hermitian, one has

$$
\mathcal{E}(a)=\mathcal{E}(0)+\operatorname{Re}\left(\mathcal{K}\left(\boldsymbol{u}^{\mathrm{N}}-\boldsymbol{u}^{\mathrm{D}}, \overline{\boldsymbol{v}}_{a}^{\mathrm{N}}-\overline{\boldsymbol{v}}_{a}^{\mathrm{D}}\right)\right)+\frac{1}{2} \mathcal{K}\left(\boldsymbol{v}_{a}^{\mathrm{N}}-\boldsymbol{v}_{a}^{\mathrm{D}}, \overline{\boldsymbol{v}}_{a}^{\mathrm{N}}-\overline{\boldsymbol{v}}_{a}^{\mathrm{D}}\right)
$$

Lemma 2.1 Let the adjoint solutions $\boldsymbol{w}^{N}, \boldsymbol{w}^{D}$ be defined by the weak formulations

$$
\begin{aligned}
& \text { find } \boldsymbol{w}^{D} \in \mathcal{V}_{D, 0}, \quad \mathcal{Z}\left(\boldsymbol{w}^{D}, \boldsymbol{w}\right)=\mathcal{K}\left(\boldsymbol{u}^{D}-\boldsymbol{u}^{N}, \boldsymbol{w}\right) \quad\left(\forall \boldsymbol{w} \in \mathcal{V}_{D, 0}\right) \\
& \text { find } \boldsymbol{w}^{N} \in \mathcal{V}_{N, 0}, \quad \mathcal{Z}\left(\boldsymbol{w}^{N}, \boldsymbol{w}\right)=\mathcal{K}\left(\boldsymbol{u}^{N}-\boldsymbol{u}^{D}, \boldsymbol{w}\right) \quad\left(\forall \boldsymbol{w} \in \mathcal{V}_{N, 0}\right)
\end{aligned}
$$

Then, $\mathcal{E}(a)$ is given by

$$
\begin{aligned}
\mathcal{E}(a)=\mathcal{E}(0)-\Re\left[\Delta \mathcal{Z}_{a}\left(\boldsymbol{w}^{N}, \overline{\boldsymbol{u}}_{a}^{N}\right)+\Delta \mathcal{Z}_{a}\left(\boldsymbol{w}^{D}, \overline{\boldsymbol{u}}_{a}^{D}\right)\right] \\
+\frac{1}{2}\left(\omega^{2} \mathcal{M}\left(\boldsymbol{v}_{a}^{N}-\boldsymbol{v}_{a}^{D}, \overline{\boldsymbol{v}}_{a}^{N}-\overline{\boldsymbol{v}}_{a}^{D}\right)-\Delta \mathcal{Z}_{a}\left(\boldsymbol{u}_{a}^{N}-\boldsymbol{u}_{a}^{D}, \overline{\boldsymbol{v}}_{a}^{N}-\overline{\boldsymbol{v}}_{a}^{D}\right)\right)
\end{aligned}
$$

Proof. Reformulation (12) of $\mathcal{E}(a)$ stems from straightforward computations. First, using the weak formulations for the adjoint and perturbed fields, one has

$$
\begin{array}{rlr}
\mathcal{K}\left(\boldsymbol{u}^{\mathrm{N}}-\boldsymbol{u}^{\mathrm{D}}, \overline{\boldsymbol{v}}_{a}^{\mathrm{N}}-\overline{\boldsymbol{v}}_{a}^{\mathrm{D}}\right) & =\mathcal{K}\left(\boldsymbol{u}^{\mathrm{N}}-\boldsymbol{u}^{\mathrm{D}}, \overline{\boldsymbol{v}}_{a}^{\mathrm{N}}\right)+\mathcal{K}\left(\boldsymbol{u}^{\mathrm{D}}-\boldsymbol{u}^{\mathrm{N}}, \overline{\boldsymbol{v}}_{a}^{\mathrm{D}}\right) & \text { using }(11 \mathrm{a}, \mathrm{b}) \\
& =\mathcal{Z}\left(\boldsymbol{w}^{\mathrm{N}}, \overline{\boldsymbol{v}}_{a}^{\mathrm{N}}\right)+\mathcal{Z}\left(\boldsymbol{w}^{\mathrm{D}}, \overline{\boldsymbol{v}}_{a}^{\mathrm{D}}\right) & \\
& =-\Delta \mathcal{Z}_{a}\left(\boldsymbol{w}^{\mathrm{N}}, \overline{\boldsymbol{u}}^{\mathrm{N}}\right)-\Delta \mathcal{Z}_{a}\left(\boldsymbol{w}^{\mathrm{N}}, \overline{\boldsymbol{v}}_{a}^{\mathrm{N}}\right)-\Delta \mathcal{Z}_{a}\left(\boldsymbol{w}^{\mathrm{D}}, \overline{\boldsymbol{u}}^{\mathrm{D}}\right)-\Delta \mathcal{Z}_{a}\left(\boldsymbol{w}^{\mathrm{D}}, \overline{\boldsymbol{v}}_{a}^{\mathrm{D}}\right) & \text { using }(9 \mathrm{a}, \mathrm{b}) \\
& =-\Delta \mathcal{Z}_{a}\left(\boldsymbol{w}^{\mathrm{N}}, \overline{\boldsymbol{u}}_{a}^{\mathrm{N}}\right)-\Delta \mathcal{Z}_{a}\left(\boldsymbol{w}^{\mathrm{D}}, \overline{\boldsymbol{u}}_{a}^{\mathrm{D}}\right) &
\end{array}
$$


Then, identities

$$
\begin{aligned}
& \mathcal{K}\left(\boldsymbol{v}_{a}^{\mathrm{D}}, \overline{\boldsymbol{v}}_{a}^{\mathrm{D}}\right)=\mathcal{Z}\left(\boldsymbol{v}_{a}^{\mathrm{D}}, \overline{\boldsymbol{v}}_{a}^{\mathrm{D}}\right)+\omega^{2} \mathcal{M}\left(\boldsymbol{v}_{a}^{\mathrm{D}}, \overline{\boldsymbol{v}}_{a}^{\mathrm{D}}\right)=-\Delta \mathcal{Z}_{a}\left(\boldsymbol{u}_{a}^{\mathrm{D}}, \overline{\boldsymbol{v}}_{a}^{\mathrm{D}}\right)+\omega^{2} \mathcal{M}\left(\boldsymbol{v}_{a}^{\mathrm{D}}, \overline{\boldsymbol{v}}_{a}^{\mathrm{D}}\right), \\
& \mathcal{K}\left(\boldsymbol{v}_{a}^{\mathrm{N}}, \overline{\boldsymbol{v}}_{a}^{\mathrm{N}}\right)=\mathcal{Z}\left(\boldsymbol{v}_{a}^{\mathrm{N}}, \overline{\boldsymbol{v}}_{a}^{\mathrm{N}}\right)+\omega^{2} \mathcal{M}\left(\boldsymbol{v}_{a}^{\mathrm{N}}, \overline{\boldsymbol{v}}_{a}^{\mathrm{N}}\right)=-\Delta \mathcal{Z}_{a}\left(\boldsymbol{u}_{a}^{\mathrm{N}}, \overline{\boldsymbol{v}}_{a}^{\mathrm{N}}\right)+\omega^{2} \mathcal{M}\left(\boldsymbol{v}_{a}^{\mathrm{N}}, \overline{\boldsymbol{v}}_{a}^{\mathrm{N}}\right), \\
& \mathcal{K}\left(\boldsymbol{v}_{a}^{\mathrm{N}}, \overline{\boldsymbol{v}}_{a}^{\mathrm{D}}\right)=\mathcal{Z}\left(\boldsymbol{v}_{a}^{\mathrm{N}}, \overline{\boldsymbol{v}}_{a}^{\mathrm{D}}\right)+\omega^{2} \mathcal{M}\left(\boldsymbol{v}_{a}^{\mathrm{N}}, \overline{\boldsymbol{v}}_{a}^{\mathrm{D}}\right)=-\Delta \mathcal{Z}_{a}\left(\boldsymbol{u}_{a}^{\mathrm{N}}, \overline{\boldsymbol{v}}_{a}^{\mathrm{D}}\right)+\omega^{2} \mathcal{M}\left(\boldsymbol{v}_{a}^{\mathrm{N}}, \overline{\boldsymbol{v}}_{a}^{\mathrm{D}}\right)
\end{aligned}
$$

(which exploit weak formulations $(9 \mathrm{a}, \mathrm{b})$ ) yield, upon collecting and rearranging terms:

$$
\mathcal{K}\left(\boldsymbol{v}_{a}^{\mathrm{N}}-\boldsymbol{v}_{a}^{\mathrm{D}}, \overline{\boldsymbol{v}}_{a}^{\mathrm{N}}-\overline{\boldsymbol{v}}_{a}^{\mathrm{D}}\right)=\omega^{2} \mathcal{M}\left(\boldsymbol{v}_{a}^{\mathrm{N}}-\boldsymbol{v}_{a}^{\mathrm{D}}, \overline{\boldsymbol{v}}_{a}^{\mathrm{N}}-\overline{\boldsymbol{v}}_{a}^{\mathrm{D}}\right)-\Delta \mathcal{Z}_{a}\left(\boldsymbol{u}_{a}^{\mathrm{N}}-\boldsymbol{u}_{a}^{\mathrm{D}}, \overline{\boldsymbol{v}}_{a}^{\mathrm{N}}-\overline{\boldsymbol{v}}_{a}^{\mathrm{D}}\right)
$$

Equation (12) finally follows from substituting (13) and (14) into (10).

The goal of reformulation $(12)$ is to recast $\mathcal{E}(a)$ as much as possible in terms of the perturbation $\Delta \mathcal{Z}_{a}$ of the dynamic stiffness operator so as to facilitate the subsequent expansion w.r.t. $a$, taking advantage of the fact that the geometrical support of $\Delta \mathcal{Z}_{a}$ is $B_{a}$. Reformulation (12) of $\mathcal{E}(a)$ is exact (i.e. no expansion has yet been performed). It is also clear from (12) that $\mathcal{E}(a)$ depends on $a$ both explicitly through the support $B_{a}$ and implicitly through solutions $\boldsymbol{u}_{a}^{\mathrm{D}, \mathrm{N}}$.

As both (12) and the right-hand sides of problems (5a) defining $\boldsymbol{u}_{a}^{\mathrm{D}, \mathrm{N}}$ involve integrals over the vanishing inclusion $B_{a}$, it is convenient for the subsequent analysis to scale the position vector $\overline{\boldsymbol{\xi}} \in B_{a}$ according to:

$$
\boldsymbol{\xi}=\boldsymbol{z}+a \overline{\boldsymbol{\xi}} \quad\left(\boldsymbol{\xi} \in B_{a}, \overline{\boldsymbol{\xi}} \in \mathcal{B}\right) \text {. }
$$

In particular, this mapping transforms integrals over $B_{a}$ into integrals over $\mathcal{B}$, with the differential volume element rescaled according to

$$
\mathrm{d} V_{\xi}=a^{3} \mathrm{~d} \bar{V}_{\bar{\xi}} \quad\left(\boldsymbol{\xi} \in B_{a}, \overline{\boldsymbol{\xi}} \in \mathcal{B}\right)
$$

\subsection{Small-inclusion asymptotics of the Neumann and Dirichlet fields}

Introduce for simplicity a generic notation for the perturbed states, where $\boldsymbol{v}_{a}$ stands for either $\boldsymbol{v}_{a}^{\mathrm{D}}$ or $\boldsymbol{v}_{a}^{\mathrm{N}}$ and weak formulations $(9 \mathrm{a}, \mathrm{b})$ have the generic form (with $\mathcal{V}_{0}$ standing for either $\mathcal{V}_{\mathrm{D}, 0}$ or $\mathcal{V}_{\mathrm{N}, 0}$ )

$$
\text { find } \boldsymbol{v}_{a} \in \mathcal{V}_{0}, \quad \mathcal{Z}_{a}\left(\boldsymbol{v}_{a}, \boldsymbol{w}\right)=-\Delta \mathcal{Z}_{a}(\boldsymbol{u}, \boldsymbol{w}) \quad\left(\forall \boldsymbol{w} \in \mathcal{V}_{0}\right)
$$

Also, let the elastodynamic Green's tensor $\mathcal{G}(\boldsymbol{\xi}, \boldsymbol{x})$ be defined by

$$
\text { find } \mathcal{G}(\cdot, \boldsymbol{x}) \in \mathcal{W}_{0}^{3}, \quad \mathcal{Z}(\boldsymbol{G}(\cdot, \boldsymbol{x}), \boldsymbol{w})=\boldsymbol{w}(\boldsymbol{x}) \quad\left(\forall \boldsymbol{w} \in \mathcal{V}_{0}\right)
$$

i.e. $\mathcal{G}(\cdot, \boldsymbol{x})$ gathers the three linearly independent elastodynamic displacement fields $\mathcal{G}^{k}(\cdot, \boldsymbol{x})=\boldsymbol{e}_{k} \cdot \mathcal{G}(\cdot, \boldsymbol{x})$ $(1 \leq k \leq 3)$ resulting from unit point forces $\delta(\cdot-\boldsymbol{x}) \boldsymbol{e}_{k}$ applied at $\boldsymbol{x} \in \Omega$ along each coordinate direction $k$ and fulfilling the homogeneous boundary conditions on $S$ implied by the definition of $\mathcal{V}_{0}$ (the clause $\mathcal{G}(\cdot, \boldsymbol{x}) \in \mathcal{W}_{0}^{3}$ symbolizing the latter condition in $(18)$, as $\mathcal{G}^{k}(\cdot, \boldsymbol{x}) \notin H^{1}(\Omega)$ due to its singular behavior of $\mathcal{G}(\cdot, \boldsymbol{x})$ at $\boldsymbol{x})$. Moreover, the ensuing analysis will be facilitated by splitting the elastodynamic Green's tensor according to

$$
\mathcal{G}(\boldsymbol{\xi}, \boldsymbol{x})=\boldsymbol{G}(\boldsymbol{\xi}-\boldsymbol{x})+\boldsymbol{G}_{\mathrm{C}}(\boldsymbol{\xi}, \boldsymbol{x})
$$

where $\boldsymbol{G}$ is the (singular) elastostatic full-space Green's tensor (i.e. Kelvin's solution) and the complementary Green's tensor $\boldsymbol{G}_{\mathrm{C}}$ is smooth at $\boldsymbol{\xi}=\boldsymbol{x}$. Importantly for the present purposes, Kelvin's solution is a homogeneous function of $\boldsymbol{r}=\boldsymbol{\xi}-\boldsymbol{x}$ :

$$
\boldsymbol{G}(\lambda \boldsymbol{r})=|\lambda|^{-1} \boldsymbol{G}(\boldsymbol{r}), \quad \boldsymbol{\nabla} \boldsymbol{G}(\lambda \boldsymbol{r})=|\lambda|^{-2} \boldsymbol{\nabla} \boldsymbol{G}(\boldsymbol{r}) \operatorname{sgn}(\lambda), \quad \lambda \in \mathbb{R}
$$

Setting $\boldsymbol{w}=\mathcal{G}(\cdot, \boldsymbol{x})$ in (17) and using the weak formulation (18), the total field $\boldsymbol{u}_{a}=\boldsymbol{u}+\boldsymbol{v}_{a}$ is found to satisfy

$$
\boldsymbol{u}_{a}(\boldsymbol{x})+\Delta \mathcal{Z}_{a}\left(\boldsymbol{u}_{a}, \mathcal{G}(\cdot, \boldsymbol{x})\right)=\boldsymbol{u}(\boldsymbol{x}) \quad(\forall \boldsymbol{x} \in \Omega)
$$

If $\boldsymbol{x} \in B_{a}$, equation (21) is readily recognized as the Lippmann-Schwinger domain integral equation governing the elastodynamic inclusion problem [24,29], written in compact form. If $\boldsymbol{x} \in \Omega \backslash \bar{B}_{a}$, equation (21) constitutes an explicit integral representation formula for $\boldsymbol{u}_{a}$ outside of the small inclusion. 
Now, guided by earlier studies $[2,18]$ which show in particular that $\boldsymbol{v}_{a}=O(a)$ in $B_{a}$, consider the following ansatz for $\boldsymbol{u}_{a}$ inside $B_{a}$ :

$$
\boldsymbol{u}_{a}(\boldsymbol{x})=\boldsymbol{u}(\boldsymbol{x})+a \boldsymbol{V}_{1}(\overline{\boldsymbol{x}})+a^{2} \boldsymbol{V}_{2}(\overline{\boldsymbol{x}})+O\left(a^{3}\right), \quad \boldsymbol{x} \in B_{a}, \overline{\boldsymbol{x}} \in \mathcal{B}
$$

Lemma 2.2 The functions $\boldsymbol{V}_{1}, \boldsymbol{V}_{2}$ featured in ansatz (22) are governed by the integral equations

$$
\begin{aligned}
& \boldsymbol{V}_{1}(\overline{\boldsymbol{x}})+\Delta \overline{\mathcal{K}}\left(\boldsymbol{V}_{1}, \boldsymbol{G}(\cdot-\overline{\boldsymbol{x}})\right)=-\Delta \overline{\mathcal{K}}\left(\boldsymbol{U}_{1}, \boldsymbol{G}(\cdot-\overline{\boldsymbol{x}})\right), \\
& \boldsymbol{V}_{2}(\overline{\boldsymbol{x}})+\Delta \overline{\mathcal{K}}\left(\boldsymbol{V}_{1}, \boldsymbol{G}(\cdot-\overline{\boldsymbol{x}})\right)=-\Delta \overline{\mathcal{K}}\left(\boldsymbol{U}_{2}, \boldsymbol{G}(\cdot-\boldsymbol{x})\right)+\omega^{2} \Delta \overline{\mathcal{M}}(\boldsymbol{u}(\boldsymbol{z}), \boldsymbol{G}(\cdot-\boldsymbol{x})),
\end{aligned}
$$

with $\boldsymbol{U}_{1}(\overline{\boldsymbol{\xi}})=\nabla \boldsymbol{u}(\boldsymbol{z}) \cdot \overline{\boldsymbol{\xi}}, 2 \boldsymbol{U}_{2}(\overline{\boldsymbol{\xi}})=\nabla^{2} \boldsymbol{u}(\boldsymbol{z}):(\overline{\boldsymbol{\xi}} \otimes \overline{\boldsymbol{\xi}})$ and where $\Delta \overline{\mathcal{K}}, \Delta \overline{\mathcal{M}}$ are the stiffness and mass perturbation operators expressed in normalized coordinates, i.e.

$$
\Delta \overline{\mathcal{K}}(\boldsymbol{u}, \boldsymbol{w})=\int_{\mathcal{B}} \boldsymbol{\nabla} \boldsymbol{u}(\overline{\boldsymbol{\xi}}): \Delta \mathcal{C}: \nabla \boldsymbol{w}(\overline{\boldsymbol{\xi}}) d \bar{V}_{\bar{\xi}}, \quad \Delta \overline{\mathcal{M}}(\boldsymbol{u}, \boldsymbol{w})=\int_{\mathcal{B}} \Delta \rho \boldsymbol{u}(\overline{\boldsymbol{\xi}}) \cdot \boldsymbol{w}(\overline{\boldsymbol{\xi}}) d \bar{V}_{\bar{\xi}}
$$

Moreover, $\boldsymbol{V}_{1}$ admits the representation

$$
\boldsymbol{V}_{1}(\overline{\boldsymbol{x}})=\boldsymbol{\nabla} \boldsymbol{u}(\boldsymbol{z}): \mathcal{V}_{1}(\overline{\boldsymbol{x}})
$$

with the third-order tensor field $\mathcal{V}_{1}(\overline{\boldsymbol{x}})$ governed by the tensorial integral equation

$$
\mathcal{V}_{1}(\overline{\boldsymbol{x}})+\Delta \overline{\mathcal{K}}\left(\mathcal{V}_{1}, \boldsymbol{G}(\cdot-\boldsymbol{x})\right)=-\Delta \overline{\mathcal{K}}(\boldsymbol{\mathcal { I }} \cdot \overline{\boldsymbol{\xi}}, \boldsymbol{G}(\cdot-\boldsymbol{x}))
$$

$\mathcal{I}$ denoting the fourth-order identity tensor such that $\mathcal{I}: \boldsymbol{A}=\boldsymbol{A}$ for any second-order tensor $\boldsymbol{A}$.

Proof. The proof is based on an expansion about $a=0$ of the Lippmann-Schwinger integral equation (21). This task mainly involves setting up the corresponding expansion of $\Delta \mathcal{Z}_{a}\left(\boldsymbol{u}_{a}, \mathcal{G}(\cdot, \boldsymbol{x})\right)$. Using decomposition (19) of the elastodynamic Green's tensor, performing the change of coordinates (15) for both $\boldsymbol{x}$ and $\boldsymbol{\xi}$, invoking the homogeneity property (20) of Kelvin's solution and the smoothness of $\boldsymbol{G}_{\mathrm{C}}(\boldsymbol{\xi}, \boldsymbol{x})$ at $\boldsymbol{x}=\boldsymbol{\xi}=\boldsymbol{z}$, the following expansions are obtained:

$$
\mathcal{G}(\boldsymbol{\xi}, \boldsymbol{x})=\frac{1}{a} \boldsymbol{G}(\overline{\boldsymbol{\xi}}-\overline{\boldsymbol{x}})+O(1), \quad \boldsymbol{\nabla}_{1} \mathcal{G}(\boldsymbol{\xi}, \boldsymbol{x})=\frac{1}{a^{2}} \boldsymbol{\nabla} \boldsymbol{G}(\overline{\boldsymbol{\xi}}-\overline{\boldsymbol{x}})+O(1)
$$

(where $\nabla_{1}$ denotes the gradient w.r.t. the first argument). On substituting (27) and ansatz (22) into $\Delta \mathcal{Z}_{a}\left(\boldsymbol{u}_{a}, \mathcal{G}(\cdot, \boldsymbol{x})\right)$ as given by (6) with $B=B_{a}$, invoking scaling (16), noting that

$$
\boldsymbol{\nabla} \boldsymbol{u}_{a}(\boldsymbol{\xi})=\nabla \boldsymbol{U}_{1}(\overline{\boldsymbol{\xi}})+\nabla \boldsymbol{V}_{1}(\overline{\boldsymbol{\xi}})+a\left[\nabla \boldsymbol{U}_{2}(\overline{\boldsymbol{\xi}})+\nabla \boldsymbol{V}_{2}(\overline{\boldsymbol{\xi}})\right]+o(a) \quad\left(\boldsymbol{\xi} \in B_{a}, \overline{\boldsymbol{\xi}} \in \mathcal{B}\right)
$$

with $\boldsymbol{U}_{1}$ and $\boldsymbol{U}_{2}$ defined as in this Lemma's statement, and gathering all contributions with like powers of $a$, one finds

$$
\begin{aligned}
\Delta \mathcal{Z}_{a}\left(\boldsymbol{u}_{a}, \mathcal{G}(\cdot, \boldsymbol{x})\right)=a \Delta \overline{\mathcal{K}}\left(\boldsymbol{V}_{1}\right. & \left.+\boldsymbol{U}_{1}, \boldsymbol{G}(\cdot-\overline{\boldsymbol{x}})\right) \\
& +a^{2}\left\{\Delta \overline{\mathcal{K}}\left(\boldsymbol{V}_{2}+\boldsymbol{U}_{2}, \boldsymbol{G}(\cdot-\overline{\boldsymbol{x}})\right)-\omega^{2} \Delta \overline{\mathcal{M}}(\boldsymbol{u}(\boldsymbol{z}), \boldsymbol{G}(\cdot-\overline{\boldsymbol{x}}))\right\}+o\left(a^{2}\right)
\end{aligned}
$$

Integral equations $(23 \mathrm{a}, \mathrm{b})$ finally result from substituting (22) and (28) into (21) and separately setting to zero the $O(a)$ and $O\left(a^{2}\right)$ contributions in the resulting equality.

Then, representation (25) and its governing equation (26) follow from $\boldsymbol{U}_{1}(\overline{\boldsymbol{\xi}})=\boldsymbol{\nabla} \boldsymbol{u}(\boldsymbol{z}): \mathcal{U}_{1}(\overline{\boldsymbol{\xi}})$ and the linearity of equation (23a).

Proposition 2.3 (outer and inner expansions of $\boldsymbol{v}_{a}$ ) The perturbed field admits the outer expansion

$$
\boldsymbol{v}_{a}(\boldsymbol{x})=a^{3}|\mathcal{B}|\left\{\boldsymbol{\nabla} \boldsymbol{u}(\boldsymbol{z}): \mathcal{A}: \nabla_{1} \mathcal{G}(\boldsymbol{z}, \boldsymbol{x})+\Delta \rho \omega^{2} \boldsymbol{u}(\boldsymbol{z}) \cdot \mathcal{G}(\boldsymbol{z}, \boldsymbol{x})\right\}+o\left(a^{3}\right)
$$


(valid for any fixed location $\boldsymbol{x} \neq \boldsymbol{z}$ ), with the polarization tensor $\mathcal{A}$ given by

$$
\mathcal{A}=-\frac{1}{|\mathcal{B}|} \int_{\mathcal{B}}(\mathcal{I}+\nabla \mathcal{V}(\overline{\boldsymbol{\xi}})): \Delta \mathcal{C} d \bar{V}_{\bar{\xi}}
$$

and the inner expansion

$$
\boldsymbol{v}_{a}(\boldsymbol{x})=a \boldsymbol{V}_{1}(\overline{\boldsymbol{x}})+a^{2} \boldsymbol{V}_{2}(\overline{\boldsymbol{x}})+O\left(a^{3}\right)
$$

(valid for any fixed $\overline{\boldsymbol{x}}$, i.e. at any location that remains close to $B_{a}$ as $a \rightarrow 0$ ), where $\boldsymbol{V}_{1}$ and $\boldsymbol{V}_{2}$ away from $\mathcal{B}$ are given by the representation formulas

$$
\begin{aligned}
& \boldsymbol{V}_{1}(\overline{\boldsymbol{x}})=-\Delta \overline{\mathcal{K}}\left(\boldsymbol{V}_{1}+\boldsymbol{U}_{1}, \boldsymbol{G}(\cdot-\overline{\boldsymbol{x}})\right), \\
& \boldsymbol{V}_{2}(\overline{\boldsymbol{x}})=-\Delta \overline{\mathcal{K}}\left(\boldsymbol{V}_{2}+\boldsymbol{U}_{2}, \boldsymbol{G}(\cdot-\overline{\boldsymbol{x}})\right)+\omega^{2} \Delta \overline{\mathcal{M}}(\boldsymbol{u}(\boldsymbol{z}), \boldsymbol{G}(\cdot-\overline{\boldsymbol{x}})),
\end{aligned}
$$

with $\boldsymbol{U}_{1}, \boldsymbol{U}_{2}$ defined as in Lemma 2.2 and where $\left.\boldsymbol{V}_{1}\right|_{\mathcal{B}},\left.\boldsymbol{V}_{2}\right|_{\mathcal{B}}$ are the solutions to equations (23a,b)

Proof. The outer expansion is obtained by studying the limiting form as $a \rightarrow 0$ of (21) considered as an integral representation for given $\boldsymbol{x} \neq \boldsymbol{z}$ with $a<\|\boldsymbol{x}-\boldsymbol{z}\|$. Performing scaling (15) and (16) and substituting ansatz (22) into (21), noting that expansions

$$
\mathcal{G}(\boldsymbol{\xi}, \boldsymbol{x})=\mathcal{G}(\boldsymbol{z}, \boldsymbol{x})+o(1), \quad \boldsymbol{\nabla}_{1} \mathcal{G}(\boldsymbol{\xi}, \boldsymbol{x})=\nabla_{1} \mathcal{G}(\boldsymbol{z}, \boldsymbol{x})+o(1)
$$

hold for $\boldsymbol{x} \neq \boldsymbol{z}$, one obtains

$$
\boldsymbol{v}_{a}(\boldsymbol{x})=-a^{3}\left\{\int_{\mathcal{B}}\left(\boldsymbol{\nabla} \boldsymbol{u}(\boldsymbol{\xi})+\nabla \boldsymbol{V}_{1}(\overline{\boldsymbol{\xi}})\right): \Delta \mathcal{C} \mathrm{d} \bar{V}_{\bar{\xi}}\right\}: \nabla_{1} \mathcal{G}(\boldsymbol{z}, \boldsymbol{x})-a^{3}\left\{\int_{\mathcal{B}} \Delta \rho \boldsymbol{u}(\boldsymbol{\xi}) \mathrm{d} \bar{V}_{\bar{\xi}}\right\} \cdot \mathcal{G}(\boldsymbol{z}, \boldsymbol{x})+o\left(a^{3}\right)
$$

Upon inserting representation (25) and expansions

$$
\boldsymbol{u}(\boldsymbol{\xi})=\boldsymbol{u}(\boldsymbol{z}+a \overline{\boldsymbol{\xi}})=\boldsymbol{u}(\boldsymbol{z})+o(1), \quad \boldsymbol{\nabla} \boldsymbol{u}(\boldsymbol{\xi})=\boldsymbol{\nabla} \boldsymbol{u}(\boldsymbol{z}+a \overline{\boldsymbol{\xi}})=\boldsymbol{\nabla} \boldsymbol{u}(\boldsymbol{z})+o(1)
$$

in (33), and noting that

$$
\int_{\mathcal{B}}\left(\boldsymbol{\nabla} \boldsymbol{u}(\boldsymbol{\xi})+\nabla \boldsymbol{V}_{1}(\overline{\boldsymbol{\xi}})\right): \Delta \mathcal{C} \mathrm{d} \bar{V}_{\bar{\xi}}=|\mathcal{B}| \nabla \boldsymbol{u}(\boldsymbol{z}): \mathcal{A}+o(1), \quad \int_{\mathcal{B}} \Delta \rho \boldsymbol{u}(\boldsymbol{\xi}) \mathrm{d} \bar{V}_{\bar{\xi}}=|\mathcal{B}| \Delta \rho \boldsymbol{u}(\boldsymbol{z})+o(1)
$$

with $\mathcal{A}$ defined by (30), the desired outer expansion (29) follows.

Next, the inner expansion is obtained by studying the limiting form as $a \rightarrow 0$ of (21) considered as an integral representation evaluated at $\boldsymbol{x}=\boldsymbol{z}+a \overline{\boldsymbol{x}}$ for given $\overline{\boldsymbol{x}} \notin \mathcal{B}$. Again, the expansion of $\Delta \mathcal{Z}_{a}\left(\boldsymbol{u}_{a}, \mathcal{G}(\cdot, \boldsymbol{x})\right)$ is required (this time for fixed $\overline{\boldsymbol{x}} \notin \overline{\mathcal{B}}$ ). This expansion is derived as in the proof of Proposition 2.3 and is still given by (28). Substituting (28) into (21) and setting $\boldsymbol{u}_{a}(\boldsymbol{x})=\boldsymbol{u}(\boldsymbol{x})+\boldsymbol{v}_{a}(\boldsymbol{x})$ finally yields the sought inner expansion (31), (32a,b).

Remark 1 The leading term $\boldsymbol{V}_{1}$ and the tensor $\mathcal{A}$ are already investigated (with different notations and using an analysis based on coupled boundary integral equations) in [2] and [18]. Moreover, the tensor $\mathcal{A}$ has major symmetry, i.e. $\mathcal{A}=\mathcal{A}^{T}$ [2].

Remark 2 For ellipsoidal inclusions, $\boldsymbol{\nabla} \mathcal{V}_{1}$ is constant inside $\mathcal{B}$ [27]. The following explicit expressions of $\mathcal{V}_{1}$ and $\mathcal{A}$ are available [18], where $\mathcal{S}=\mathcal{S}(\mathcal{B})$ denotes the Eshelby tensor for the normalized ellipsoid $\mathcal{B}$ (given in explicit form in [27]):

$$
\text { (a) } \nabla \mathcal{V}(\overline{\boldsymbol{\xi}})=-\Delta \mathcal{C}:\left[\mathcal{C}+\mathcal{S}^{T}: \Delta \mathcal{C}\right]^{-1}: \mathcal{S}^{T}, \quad \text { (b) } \mathcal{A}=-\Delta \mathcal{C}:\left[\mathcal{C}+\mathcal{S}^{T}: \Delta \mathcal{C}\right]^{-1}: \mathcal{C}
$$

These results also hold for the special case of a traction-free cavity, with $\rho^{\star}=0$ and $\mathcal{C}^{\star}=\mathbf{0}$ (i.e. $\Delta \mathcal{C}=-\mathcal{C}$ ).

Remark 3 Inner, outer and uniform small-inclusion asymptotics of time-harmonic electromagnetic fields are established in [3].

A uniform expansion of $\boldsymbol{v}_{a}$ may then be obtained by the method of matched asymptotic expansions, see e.g. [26] or [3], whereby the outer expansion (29) and the inner expansion (31) are "blended" by enforcing 
their coincidence at evaluation points $\boldsymbol{x}=\boldsymbol{z}+a^{\alpha} \hat{\boldsymbol{x}}=\boldsymbol{x}_{\alpha}$ (with $0<\alpha<1$ ) which are "intermediate" in that $\left\|\boldsymbol{x}_{\alpha}-\boldsymbol{z}\right\| \rightarrow 0$ while $\left\|\boldsymbol{x}_{\alpha}-\boldsymbol{z}\right\| / a \rightarrow \infty$ as $a \rightarrow 0$. Here, the following direct consequence of (29) and (31) is sufficient for the present purposes, while a proper matching of (29) and (31) would yield the detailed form of the $O\left(a^{3}\right)$ contribution.

Proposition 2.4 (uniform expansion of $\boldsymbol{v}_{a}$ ) The perturbed field $\boldsymbol{v}_{a}$ admits the uniform expansion

$$
\boldsymbol{v}_{a}(\boldsymbol{x})=a \boldsymbol{V}_{1}\left(\frac{\boldsymbol{x}-\boldsymbol{z}}{a}\right)+a^{2} \boldsymbol{V}_{2}\left(\frac{\boldsymbol{x}-\boldsymbol{z}}{a}\right)+O\left(a^{3}\right)
$$

where $\boldsymbol{V}_{1}, \boldsymbol{V}_{2}$ are defined by $(32 a, b)$.

\subsection{Topological sensitivity of energy cost functional}

Lemma 2.5 One has

$$
\begin{aligned}
\Delta \mathcal{Z}_{a}\left(\overline{\boldsymbol{u}}_{a}^{D}, \boldsymbol{w}^{D}\right) & =-a^{3}|\mathcal{B}|\left[\nabla \boldsymbol{w}^{D}: \mathcal{A}: \nabla \overline{\boldsymbol{u}}^{D}+\Delta \rho \omega^{2} \boldsymbol{w}^{D} \cdot \overline{\boldsymbol{u}}^{D}\right](\boldsymbol{z})+o\left(a^{3}\right) \\
\Delta \mathcal{Z}_{a}\left(\overline{\boldsymbol{u}}_{a}^{N}, \boldsymbol{w}^{N}\right) & =-a^{3}|\mathcal{B}|\left[\boldsymbol{\nabla} \boldsymbol{w}^{N}: \mathcal{A}: \boldsymbol{\nabla} \overline{\boldsymbol{u}}^{N}+\Delta \rho \omega^{2} \boldsymbol{w}^{D} \cdot \overline{\boldsymbol{u}}^{D}\right](\boldsymbol{z})+o\left(a^{3}\right) \\
\Delta \mathcal{Z}_{a}\left(\boldsymbol{u}_{a}^{N}-\boldsymbol{u}_{a}^{D}, \overline{\boldsymbol{v}}_{a}^{N}-\overline{\boldsymbol{v}}_{a}^{D}\right) & =-2 a^{3}|\mathcal{B}|\left[\boldsymbol{\nabla}\left(\boldsymbol{u}^{N}-\boldsymbol{u}^{D}\right): \mathcal{B}: \boldsymbol{\nabla}\left(\overline{\boldsymbol{u}}^{N}-\overline{\boldsymbol{u}}^{D}\right)\right](\boldsymbol{z})+o\left(a^{3}\right)
\end{aligned}
$$

where the fourth-order tensor $\mathcal{B}$ is defined by

$$
\mathcal{B}=-\frac{1}{2|\mathcal{B}|} \int_{\mathcal{B}}\left(\mathcal{I}+\nabla \mathcal{V}_{1}\right): \Delta \mathcal{C}: \nabla \mathcal{V}_{1}^{T} d \bar{V}_{\bar{\xi}}
$$

Proof. To establish the $O\left(a^{3}\right)$ expansions (36a-c), one notes from scaling (16) that this task amounts to seeking the leading $O(1)$ contributions yielded by expanding the densities of the respective integrals. The latter are readily found by noting that

$$
\begin{aligned}
\boldsymbol{u}^{\mathrm{D}, \mathrm{N}}(\boldsymbol{x}) & =\boldsymbol{u}^{\mathrm{D}, \mathrm{N}}(\boldsymbol{z})+o(1), & \boldsymbol{w}^{\mathrm{D}, \mathrm{N}}(\boldsymbol{x}) & =\boldsymbol{w}^{\mathrm{D}, \mathrm{N}}(\boldsymbol{z})+o(1), \\
\boldsymbol{\nabla} \boldsymbol{u}^{\mathrm{D}, \mathrm{N}}(\boldsymbol{x}) & =\boldsymbol{\nabla} \boldsymbol{u}^{\mathrm{D}, \mathrm{N}}(\boldsymbol{z})+o(1), & \boldsymbol{\nabla} \boldsymbol{w}^{\mathrm{D}, \mathrm{N}}(\boldsymbol{x}) & =\boldsymbol{\nabla} \boldsymbol{w}^{\mathrm{D}, \mathrm{N}}(\boldsymbol{z})+o(1),
\end{aligned}
$$

which stem from the smoothness of the free and adjoint fields at $\boldsymbol{z}$, and

$$
\begin{aligned}
\boldsymbol{u}_{a}^{\mathrm{D}, \mathrm{N}}(\boldsymbol{x}) & =\boldsymbol{u}^{\mathrm{D}, \mathrm{N}}(\boldsymbol{z})+o(1), & \boldsymbol{v}_{a}^{\mathrm{D}, \mathrm{N}}(\boldsymbol{x}) & =o(1) \\
\boldsymbol{\nabla} \boldsymbol{u}_{a}^{\mathrm{D}, \mathrm{N}}(\boldsymbol{x}) & =\boldsymbol{\nabla} \boldsymbol{u}^{\mathrm{D}, \mathrm{N}}(\boldsymbol{z}):\left(\mathcal{I}+\nabla \mathcal{V}_{1}(\overline{\boldsymbol{\xi}})\right)+o(1), & \boldsymbol{\nabla} \boldsymbol{v}_{a}^{\mathrm{D}, \mathrm{N}}(\boldsymbol{x}) & =\boldsymbol{\nabla} \boldsymbol{u}^{\mathrm{D}, \mathrm{N}}: \nabla \mathcal{V}_{1}(\overline{\boldsymbol{\xi}})+o(1)
\end{aligned}
$$

which result from the asymptotic behavior of $\boldsymbol{u}_{a}=\boldsymbol{u}+\boldsymbol{v}_{a}$ established in Lemma 2.2.

First, using (38a-d) in (36a), one obtains

$$
\begin{aligned}
\Delta \mathcal{Z}_{a}\left(\boldsymbol{u}_{a}^{\mathrm{D}}, \overline{\boldsymbol{w}}^{\mathrm{D}}\right)= & a^{3} \int_{\mathcal{B}}\left[\left(\boldsymbol{\nabla} \boldsymbol{u}^{\mathrm{D}}(\boldsymbol{z}):\left(\mathcal{I}+\nabla \mathcal{V}_{1}(\overline{\boldsymbol{\xi}})\right)+o(1)\right): \Delta \mathcal{C}:\left(\boldsymbol{\nabla} \overline{\boldsymbol{w}}^{\mathrm{D}}(\boldsymbol{z})+o(1)\right)\right. \\
& \left.+\Delta \rho \omega^{2}\left(\boldsymbol{u}^{\mathrm{D}}(\boldsymbol{z})+o(1)\right) \cdot\left(\overline{\boldsymbol{w}}^{\mathrm{D}}(\boldsymbol{z})+o(1)\right)\right] \mathrm{d} \bar{V}_{\bar{\xi}} \\
= & a^{3}\left[\boldsymbol{\nabla} \boldsymbol{u}^{\mathrm{D}}(\boldsymbol{z}):\left\{\int_{\mathcal{B}}\left(\mathcal{I}+\nabla \mathcal{V}_{1}(\overline{\boldsymbol{\xi}})\right): \Delta \mathcal{C} \mathrm{d} \bar{V}_{\bar{\xi}}\right\}: \nabla \overline{\boldsymbol{w}}^{\mathrm{D}}(\boldsymbol{z})+|\mathcal{B}| \Delta \rho \omega^{2} \boldsymbol{u}^{\mathrm{D}}(\boldsymbol{z}) \cdot \overline{\boldsymbol{w}}^{\mathrm{D}}(\boldsymbol{z})\right]+o\left(a^{3}\right)
\end{aligned}
$$

Inserting definition (30) of $\mathcal{A}$ in the last equality above then yields expansion (36a), with expansion (36b) obtained in exactly the same way by replacing all "Dirichlet" fields by "Neumann" fields.

Similarly, using $(38 \mathrm{a}-\mathrm{d})$ in $(36 \mathrm{c})$ gives

$$
\begin{aligned}
& \Delta \mathcal{Z}_{a}\left(\boldsymbol{u}_{a}^{\mathrm{N}}-\boldsymbol{u}_{a}^{\mathrm{D}}, \overline{\boldsymbol{v}}_{a}^{\mathrm{N}}-\overline{\boldsymbol{v}}_{a}^{\mathrm{D}}\right) \\
& =a^{3} \int_{\mathcal{B}}\left[\left(\boldsymbol{\nabla}\left(\boldsymbol{u}^{\mathrm{N}}-\boldsymbol{u}^{\mathrm{D}}\right)(\boldsymbol{z}):\left(\mathcal{I}+\boldsymbol{\nabla} \mathcal{V}_{1}(\overline{\boldsymbol{\xi}})\right)+o(1)\right): \Delta \mathcal{C}:\left(\boldsymbol{\nabla}\left(\overline{\boldsymbol{u}}^{\mathrm{N}}-\overline{\boldsymbol{u}}^{\mathrm{D}}\right)(\boldsymbol{z}): \nabla \mathcal{V}_{1}(\overline{\boldsymbol{\xi}})+o(1)\right)\right. \\
& \left.+\Delta \rho \omega^{2}\left(\left(\boldsymbol{u}^{\mathrm{N}}-\boldsymbol{u}^{\mathrm{D}}\right)(\boldsymbol{z})+o(1)\right) \cdot o(1)\right] \mathrm{d} \bar{V}_{\bar{\xi}}
\end{aligned}
$$




$$
=a^{3} \boldsymbol{\nabla}\left(\boldsymbol{u}^{\mathrm{N}}-\boldsymbol{u}^{\mathrm{D}}\right)(\boldsymbol{z}):\left\{\int_{\mathcal{B}}\left(\mathcal{I}+\nabla \mathcal{V}_{1}(\overline{\boldsymbol{\xi}})\right): \Delta \mathcal{C}: \boldsymbol{\nabla} \mathcal{V}_{1}^{\mathrm{T}}(\overline{\boldsymbol{\xi}}) \mathrm{d} \bar{V}_{\bar{\xi}}\right\}: \nabla\left(\overline{\boldsymbol{u}}^{\mathrm{N}}-\overline{\boldsymbol{u}}^{\mathrm{D}}\right)(\boldsymbol{z})+o\left(a^{3}\right)
$$

Upon expressing the bracketed integral in terms of $\mathcal{B}$ defined by (37), the above equality becomes the sought result $(36 \mathrm{c})$.

Remark 4 For ellipsoidal inclusions, $\mathcal{B}$ is found, using (34), to be given by $2 \mathcal{B}=-\mathcal{A}: \mathcal{S}: \mathcal{C}^{-1}: \mathcal{A}$.

Lemma 2.6 One has

$$
\mathcal{M}\left(\boldsymbol{v}_{a}^{N}-\boldsymbol{v}_{a}^{D}, \overline{\boldsymbol{v}}_{a}^{N}-\overline{\boldsymbol{v}}_{a}^{D}\right)=O\left(a^{4}\right)
$$

Proof. The task at hand is to evaluate the leading contribution as $a \rightarrow 0$ of the integral

$$
\mathcal{M}\left(\boldsymbol{v}_{a}^{\mathrm{N}}-\boldsymbol{v}_{a}^{\mathrm{D}}, \boldsymbol{v}_{a}^{\mathrm{N}}-\boldsymbol{v}_{a}^{\mathrm{D}}\right)=\int_{\Omega} \rho\left(\boldsymbol{v}_{a}^{\mathrm{N}}-\boldsymbol{v}_{a}^{\mathrm{D}}\right) \cdot\left(\overline{\boldsymbol{v}}_{a}^{\mathrm{N}}-\overline{\boldsymbol{v}}_{a}^{\mathrm{D}}\right) \mathrm{d} V
$$

and more specifically to establish that this leading contribution is $o\left(a^{3}\right)$. This is not immediately obvious as $\boldsymbol{v}_{a}^{\mathrm{N}}-\boldsymbol{v}_{a}^{\mathrm{D}}$ is, by virtue of Lemma 2.3, of order $O\left(a^{3}\right)$ at any fixed location away from $B_{a}$ but of order $O(a)$ in the vicinity of $B_{a}$. Replacing $\boldsymbol{v}_{a}^{\mathrm{N}}-\boldsymbol{v}_{a}^{\mathrm{D}}$ in the above integral with expansion (35) wherein $\boldsymbol{V}_{1}, \boldsymbol{V}_{2}$ are defined according to Lemma 2.2 with $\boldsymbol{u}$ replaced with $\boldsymbol{u}^{\mathrm{N}}-\boldsymbol{u}^{\mathrm{D}}$, one readily obtains

$$
\begin{aligned}
\mathcal{M}\left(\boldsymbol{v}_{a}^{\mathrm{N}}-\boldsymbol{v}_{a}^{\mathrm{D}}, \boldsymbol{v}_{a}^{\mathrm{N}}-\boldsymbol{v}_{a}^{\mathrm{D}}\right) & =a^{2} \int_{\Omega}\left[\boldsymbol{V}_{1}\left(\frac{\boldsymbol{x}-\boldsymbol{z}}{a}\right)+a \boldsymbol{V}_{2}\left(\frac{\boldsymbol{x}-\boldsymbol{z}}{a}\right)\right] \cdot\left[\overline{\boldsymbol{V}}_{1}\left(\frac{\boldsymbol{x}-\boldsymbol{z}}{a}\right)+a \overline{\boldsymbol{V}}_{2}\left(\frac{\boldsymbol{x}-\boldsymbol{z}}{a}\right)\right] \mathrm{d} V_{x}+O\left(a^{4}\right) \\
& =a^{5} \int_{\mathcal{O}}\left[\boldsymbol{V}_{1}(\overline{\boldsymbol{x}})+a \boldsymbol{V}_{2}(\overline{\boldsymbol{x}})\right] \cdot\left[\overline{\boldsymbol{V}}_{1}(\overline{\boldsymbol{x}})+a \overline{\boldsymbol{V}}_{2}(\overline{\boldsymbol{x}})\right] \mathrm{d} \bar{V}_{\bar{x}}+O\left(a^{4}\right)
\end{aligned}
$$

where the last step stems from performing the coordinate transformation (15), (16) in the integral, with $\mathcal{O}=(\Omega-\boldsymbol{z}) / a=\mathcal{O}(\boldsymbol{z}, a)$ denoting the transformed domain of $\Omega$ through (15). However, as the transformed domain $\mathcal{O}$ depends on $a$ and becomes unbounded in the limit $a \rightarrow 0$, the behaviour as $a \rightarrow 0$ of the integral over $\mathcal{O}$ is not necessarily $O(1)$ and has to be determined.

Using representations (32a) together with the fact that the behavior of the Kelvin solution as $\|\overline{\boldsymbol{x}}\| \rightarrow \infty$ is such that $[6]$

one finds that

$$
\boldsymbol{G}(\overline{\boldsymbol{\xi}}-\overline{\boldsymbol{x}})=O\left(\|\overline{\boldsymbol{x}}\|^{-1}\right), \quad \boldsymbol{\nabla} \boldsymbol{G}(\overline{\boldsymbol{\xi}}-\overline{\boldsymbol{x}})=O\left(\|\overline{\boldsymbol{x}}\|^{-2}\right),
$$

$$
\text { (a) }\left\|\boldsymbol{V}_{1}(\overline{\boldsymbol{x}})\right\|^{2}=O\left(\|\overline{\boldsymbol{x}}\|^{-4}\right), \quad \text { (b) } \boldsymbol{V}_{1}(\overline{\boldsymbol{x}}) \cdot \overline{\boldsymbol{V}}_{2}(\overline{\boldsymbol{x}})=O\left(\|\overline{\boldsymbol{x}}\|^{-3}\right), \quad \text { (c) }\left\|\boldsymbol{V}_{2}(\overline{\boldsymbol{x}})\right\|^{2}=O\left(\|\overline{\boldsymbol{x}}\|^{-2}\right)
$$

Letting $d=d(\boldsymbol{z})$ denote the radius of the smallest sphere centered at $\boldsymbol{z}$ and enclosing $\Omega$, the transformed domain $\mathcal{O}(\boldsymbol{z}, a)$ is then enclosed in the ball of radius $d / a$ centered at the origin of the $\overline{\boldsymbol{x}}$-space. Hence, one has for example

$$
\int_{\mathcal{O}}\left\|\boldsymbol{V}_{1}(\overline{\boldsymbol{x}})\right\|^{2} \mathrm{~d} \bar{V}_{\bar{x}} \leq \int_{\|\overline{\boldsymbol{x}}\| \leq d / a}\left\|\boldsymbol{V}_{1}(\overline{\boldsymbol{x}})\right\|^{2} \mathrm{~d} \bar{V}_{\bar{x}}
$$

and (42a) together with using spherical coordinates in the above integral readily allow to show that the latter has a finite limit as $a \rightarrow 0$ (i.e. as the ball $\|\overline{\boldsymbol{x}}\| \leq d / a$ becomes unbounded), i.e. that one has

$$
\int_{\mathcal{O}}\left\|\boldsymbol{V}_{1}(\overline{\boldsymbol{x}})\right\|^{2} \mathrm{~d} \bar{V}_{\bar{x}}=O(1) \quad(a \rightarrow 0)
$$

Similar arguments allow to show that

$$
\left|\int_{\mathcal{O}} \boldsymbol{V}_{1}(\overline{\boldsymbol{x}}) \cdot \overline{\boldsymbol{V}}_{2}(\overline{\boldsymbol{x}}) \mathrm{d} \bar{V}_{\bar{x}}\right|=O(-\ln a), \quad \int_{\mathcal{O}}\left\|\boldsymbol{V}_{2}(\overline{\boldsymbol{x}})\right\|^{2} \mathrm{~d} \bar{V}_{\bar{x}}=O\left(a^{-1}\right) \quad(a \rightarrow 0)
$$

Combining these partial estimations, one obtains

$$
\int_{\mathcal{O}}\left[\boldsymbol{V}_{1}(\overline{\boldsymbol{x}})+a \boldsymbol{V}_{2}(\overline{\boldsymbol{x}})\right] \cdot\left[\overline{\boldsymbol{V}}_{1}(\overline{\boldsymbol{x}})+a \overline{\boldsymbol{V}}_{2}(\overline{\boldsymbol{x}})\right] \mathrm{d} \bar{V}_{\bar{x}}=O(1)+a O(-\ln a)+a^{2} O\left(a^{-1}\right)=O(1) .
$$

Using the above in (40) finally yields the desired expansion (39). 
Applying expansions (36a-c) and (39) to expression (12) of $\mathcal{E}(a)$, one readily finds that the leading contribution of $\mathcal{E}(a)-\mathcal{E}(0)$ is $O\left(a^{3}\right)$ and arrives at the following main result, namely the topological sensitivity of $\mathcal{E}(a)$.

Proposition 2.7 (Topological sensitivity of energy functional) The energy functional admits the expansion

with the topological sensitivity $\mathcal{T}(\boldsymbol{z})$ given by

$$
\mathcal{E}(a)=\mathcal{E}(0)+a^{3}|\mathcal{B}| \mathcal{T}(\boldsymbol{z})+o\left(a^{3}\right)
$$

$$
\begin{aligned}
\mathcal{T}(\boldsymbol{z})=\operatorname{Re}\left[\boldsymbol{\nabla} \boldsymbol{w}^{D}: \mathcal{A}: \nabla \overline{\boldsymbol{u}}^{D}+\boldsymbol{\nabla} \boldsymbol{w}^{N}: \mathcal{A}: \nabla \overline{\boldsymbol{u}}^{N}+\Delta \rho \omega^{2}\left(\boldsymbol{w}^{D} \cdot \overline{\boldsymbol{u}}^{D}+\boldsymbol{w}^{N} \cdot \overline{\boldsymbol{u}}^{N}\right)\right](\boldsymbol{z}) & +\left[\boldsymbol{\nabla}\left(\boldsymbol{u}^{N}-\boldsymbol{u}^{D}\right): \mathcal{B}: \boldsymbol{\nabla}\left(\overline{\boldsymbol{u}}^{N}-\overline{\boldsymbol{u}}^{D}\right)\right](\boldsymbol{z})
\end{aligned}
$$

\section{The acoustic case}

The approach followed in section 2 is also applicable to acoustic media, where the reference medium is characterized by its wave velocity $c$ and mass density $\rho$, while inclusions $B_{a}$ are endowed with characteristics $c^{\star}, \rho^{\star}$. The primary variable is now the scalar pressure field $u$. Retaining the notational conventions of section 2 with the necessary adaptations, the dynamic operators $\mathcal{Z}(u, w)=\mathcal{K}(u, w)-\omega^{2} \mathcal{M}(u, w)$ and $\mathcal{Z}_{a}(u, w)=\mathcal{Z}(u, w)+\Delta \mathcal{Z}_{a}(u, w)$ are now defined by

$$
\mathcal{Z}(u, w)=\int_{\Omega}\left(\boldsymbol{\nabla} u \cdot \nabla w-k^{2} u w\right) \mathrm{d} V, \quad \Delta \mathcal{Z}_{a}(u, w)=\int_{B_{a}}\left[(\beta-1) \nabla u \cdot \nabla w-\left(\beta \gamma^{2}-1\right) k^{2} u w\right] \mathrm{d} V
$$

having set $k=\omega / c, \beta=\rho / \rho^{\star}$ and $\gamma=c / c^{\star}$.

The energy cost functional $E\left(B, \rho^{\star}, c^{\star}\right)$ is next defined by

$$
E\left(B, \rho^{\star}, c^{\star}\right):=\left(\rho \omega^{2}\right)^{-1} \mathcal{K}\left(u_{a}^{\mathrm{N}}-u_{a}^{\mathrm{D}}, \bar{u}_{a}^{\mathrm{N}}-\bar{u}_{a}^{\mathrm{D}}\right)
$$

in terms of the 'Dirichlet' and 'Neumann' total fields $u_{a}^{\mathrm{D}, \mathrm{N}}$ governed by the weak formulations

$$
\begin{array}{lll}
\text { find } u_{a}^{\mathrm{N}} \in \mathcal{V}_{\mathrm{N}}, \mathcal{Z}_{a}\left(u_{a}^{\mathrm{N}}, w\right)=\mathcal{F}(w)+\mathcal{F}_{\mathrm{N}}(w) & & \left(\forall w \in \mathcal{V}_{\mathrm{N}, 0}\right), \\
\text { find } u_{a}^{\mathrm{D}} \in \mathcal{V}_{\mathrm{D}}, \mathcal{Z}_{a}\left(u_{a}^{\mathrm{D}}, w\right)=\mathcal{F}(w) & & \left(\forall w \in \mathcal{V}_{\mathrm{D}, 0}\right),
\end{array}
$$

where $\mathcal{V}_{\mathrm{N}}, \mathcal{V}_{\mathrm{N}, 0}, \mathcal{V}_{\mathrm{D}}, \mathcal{V}_{\mathrm{D}, 0}$ are now spaces of scalar functions having definitions similar to (4a,d), with the Dirichlet data $U$ in definitions $(4 \mathrm{~b}, \mathrm{~d})$ also scalar. The factor $1 / \rho \omega^{2}$ in definition (46) confers the dimensionality of an energy to $E$. In the small inclusion case, $\mathcal{E}(a)$ is defined as $\mathcal{E}(a)=E\left(B_{a}(\boldsymbol{z}), \rho^{\star}, c^{\star}\right)$. Definition (45) of $\mathcal{Z}(u, w)$ and $\Delta \mathcal{Z}_{a}(u, w)$ implies satisfaction by $u^{a}$ of the transmission condition $\llbracket \rho^{-1} \partial_{n} u^{a} \rrbracket=0$ across $\partial B_{a}$, which expresses continuity of the normal velocity.

Moreover, the adjoint solutions $w^{\mathrm{D}, \mathrm{N}}$ associated to $E$ are now defined by

$$
\begin{array}{lll}
\text { find } w^{\mathrm{D}} \in \mathcal{V}_{\mathrm{D}, 0}, & \mathcal{Z}\left(w^{\mathrm{D}}, w\right)=\left(\rho \omega^{2}\right)^{-1} \mathcal{K}\left(u^{\mathrm{D}}-u^{\mathrm{N}}, w\right) & \left(\forall w \in \mathcal{V}_{\mathrm{D}, 0}\right) \\
\text { find } w^{\mathrm{N}} \in \mathcal{V}_{\mathrm{N}}, & \mathcal{Z}\left(w^{\mathrm{N}}, w\right)=\left(\rho \omega^{2}\right)^{-1} \mathcal{K}\left(u^{\mathrm{N}}-u^{\mathrm{D}}, w\right) & \left(\forall w \in \mathcal{V}_{\mathrm{N}, 0}\right)
\end{array}
$$

The following counterpart of Lemma 2.1 allows to reformulate $\mathcal{E}(a)$ :

Lemma 3.1 Let the adjoint solutions $w^{N}, w^{D}$ be defined by $(48 a, b)$. Then, $\mathcal{E}(a)$ is given by

$$
\begin{aligned}
\mathcal{E}(a)=\mathcal{E}(0)-\Re\left[\Delta \mathcal{Z}_{a}\left(w^{N}, \bar{u}_{a}^{N}\right)+\Delta \mathcal{Z}_{a}\left(w^{D},\right.\right. & \left.\left.\bar{u}_{a}^{D}\right)\right] \\
& +\frac{1}{2}\left(\omega^{2} \mathcal{M}\left(v_{N}^{a}-v_{D}^{a}, \bar{v}_{a}^{N}-\bar{v}_{a}^{D}\right)-\Delta \mathcal{Z}_{a}\left(u_{a}^{N}-u_{a}^{D}, \bar{v}_{a}^{N}-\bar{v}_{a}^{D}\right)\right)
\end{aligned}
$$


The proof follows the same pattern as that of Lemma 2.1 and is therefore omitted.

Next, letting $\mathcal{G}$ denote the (scalar) Green's function defined by the acoustic counterpart of (18) and setting $\mathcal{G}(\boldsymbol{\xi}, \boldsymbol{x})=G(\boldsymbol{\xi}-\boldsymbol{x})+G_{\mathrm{C}}(\boldsymbol{\xi}, \boldsymbol{x})$ with $G(\boldsymbol{r})=1 /(4 \pi\|\boldsymbol{r}\|)$, the total field $u^{a}=u+v^{a}$ satisfies

$$
u^{a}(\boldsymbol{x})+\Delta \mathcal{Z}_{a}\left(u^{a}, \mathcal{G}(\cdot, \boldsymbol{x})\right)=u(\boldsymbol{x}) \quad(\forall \boldsymbol{x} \in \Omega) .
$$

and the analogue of ansatz (22) can be postulated, leading to the expected counterpart of Lemma 2.2 . The inner, outer and uniform expansions of $v^{a}$ are then found as follows:

Proposition 3.2 (inner, outer and uniform expansions of $v^{a}$ ) The perturbed field admits the inner expansion

$$
v^{a}(\boldsymbol{x})=a \boldsymbol{\nabla} u(\boldsymbol{z}) \cdot \mathcal{V}_{1}(\overline{\boldsymbol{x}})+a^{2} \boldsymbol{\nabla}^{2} u(\boldsymbol{z}): \mathcal{V}_{2}(\overline{\boldsymbol{x}})+O\left(a^{3}\right)
$$

(valid for any fixed $\overline{\boldsymbol{x}}$, i.e. at any location that remains close to $B_{a}$ as $a \rightarrow 0$ ), where $\left.\mathcal{V}_{1}\right|_{\mathcal{B}},\left.\mathcal{V}_{2}\right|_{\mathcal{B}}$ are the solutions to equations

$$
\begin{aligned}
& \mathcal{V}_{1}(\overline{\boldsymbol{x}})+\Delta \overline{\mathcal{K}}\left(\mathcal{V}_{1}, G(\cdot-\overline{\boldsymbol{x}})\right)=-\Delta \overline{\mathcal{K}}\left(\mathcal{U}_{1}, G(\cdot-\overline{\boldsymbol{x}})\right), \\
& \mathcal{V}_{2}(\overline{\boldsymbol{x}})+\Delta \overline{\mathcal{K}}\left(\mathcal{V}_{2}, G(\cdot-\overline{\boldsymbol{x}})\right)=-\Delta \overline{\mathcal{K}}\left(\mathcal{U}_{2}, G(\cdot-\boldsymbol{x})\right)-c^{2} \Delta \overline{\mathcal{M}}(\boldsymbol{I}, G(\cdot-\overline{\boldsymbol{x}})),
\end{aligned}
$$

with $\mathcal{U}_{1}(\overline{\boldsymbol{\xi}})=\overline{\boldsymbol{\xi}}, \mathcal{U}_{2}(\overline{\boldsymbol{\xi}})=(\overline{\boldsymbol{\xi}} \otimes \overline{\boldsymbol{\xi}}) / 2$, and $\mathcal{V}_{1}, \mathcal{V}_{2}$ away from $\mathcal{B}$ are given by

$$
\begin{aligned}
& \mathcal{V}_{1}(\overline{\boldsymbol{x}})=-\Delta \overline{\mathcal{K}}\left(\mathcal{V}_{1}+\mathcal{U}_{1}, G(\cdot-\overline{\boldsymbol{x}})\right), \\
& \mathcal{V}_{2}(\overline{\boldsymbol{x}})=-\Delta \overline{\mathcal{K}}\left(\mathcal{V}_{2}+\mathcal{U}_{2}, G(\cdot-\overline{\boldsymbol{x}})\right)-c^{2} \Delta \overline{\mathcal{M}}(\boldsymbol{I}, G(\cdot-\overline{\boldsymbol{x}}))
\end{aligned}
$$

Moreover, $v^{a}$ admits the outer expansion

$$
v^{a}(\boldsymbol{x})=a^{3}|\mathcal{B}|\left\{\boldsymbol{\nabla} u(\boldsymbol{z}) \cdot \boldsymbol{A} \cdot \boldsymbol{\nabla}_{1} \mathcal{G}(\boldsymbol{z}, \boldsymbol{x})+\left(\beta \gamma^{2}-1\right) k^{2} u(\boldsymbol{z}) \mathcal{G}(\boldsymbol{z}, \boldsymbol{x})\right\}+o\left(a^{3}\right)
$$

(valid for any fixed location $\boldsymbol{x} \neq \boldsymbol{z}$ ), with the second-order polarization tensor $\boldsymbol{A}$ given by

$$
\mathcal{A}=\frac{1-\beta}{|\mathcal{B}|} \int_{\mathcal{B}}(\boldsymbol{I}+\nabla \mathcal{V}(\overline{\boldsymbol{\xi}})) d \bar{V}_{\bar{\xi}}
$$

Finally, $v^{a}$ admits the uniform expansion

$$
v^{a}(\boldsymbol{x})=a \boldsymbol{\nabla} u(\boldsymbol{z}) \cdot \mathcal{V}_{1}\left(\frac{\boldsymbol{x}-\boldsymbol{z}}{a}\right)+a^{2} \boldsymbol{\nabla}^{2} u(\boldsymbol{z}): \mathcal{V}_{2}\left(\frac{\boldsymbol{x}-\boldsymbol{z}}{a}\right)+O\left(a^{3}\right)
$$

Proposition 3.3 (Topological sensitivity of energy functional (acoustic case)) The energy functional admits the expansion

$$
\mathcal{E}(a)=\mathcal{E}(0)+a^{3}|\mathcal{B}| \mathcal{T}(\boldsymbol{z})+o\left(a^{3}\right)
$$

with the topological sensitivity $\mathcal{T}(\boldsymbol{z})$ given by

$$
\begin{aligned}
& \mathcal{T}(\boldsymbol{z})=\operatorname{Re}\left[\boldsymbol{\nabla} w^{D} \cdot \boldsymbol{A} \cdot \boldsymbol{\nabla} \bar{u}^{D}+\boldsymbol{\nabla} w^{N} \cdot \boldsymbol{A} \cdot \boldsymbol{\nabla} \bar{u}^{N}+\left(\beta \gamma^{2}-1\right) k^{2}\left(w^{D} \bar{u}^{D}+w^{N} \bar{u}^{N}\right)\right](\boldsymbol{z}) \\
&+ {\left[\boldsymbol{\nabla}\left(u^{N}-u^{D}\right) \cdot \boldsymbol{B} \cdot \boldsymbol{\nabla}\left(\bar{u}^{N}-\bar{u}^{D}\right)\right](\boldsymbol{z}) }
\end{aligned}
$$

with $\boldsymbol{A}$ defined by (55) and

$$
\boldsymbol{B}=\frac{1-\beta}{2|\mathcal{B}|} \int_{\mathcal{B}}(\boldsymbol{I}+\boldsymbol{\nabla} \mathcal{V}(\overline{\boldsymbol{\xi}})) \cdot \nabla \mathcal{V}^{T}(\overline{\boldsymbol{\xi}}) d \bar{V}_{\bar{\xi}}
$$

When $B_{a}$ is a spherical inclusion, one has $\boldsymbol{\nabla} \mathcal{V}(\overline{\boldsymbol{\xi}})=(1-\beta)(2+\beta)^{-1} \boldsymbol{I}$ [17], and hence

$$
\boldsymbol{A}=\frac{3(1-\beta)}{2+\beta} \boldsymbol{I}, \quad \boldsymbol{B}=\frac{3(1-\beta)^{2}}{2(2+\beta)^{2}} \boldsymbol{I}
$$




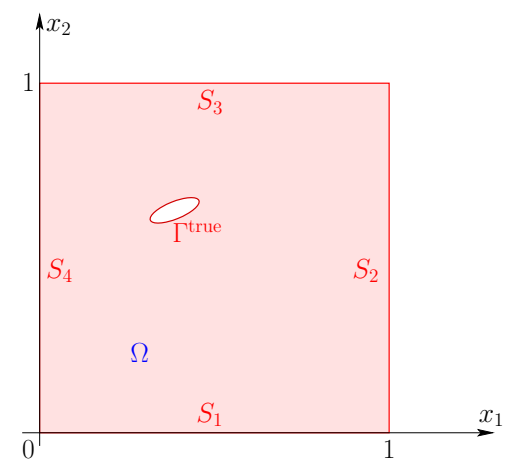

Fig. 1. Defect imaging using acoustic time-domain data: geometry and notations.

\section{Numerical example}

In this example, the reference domain $\Omega$ is the unit square, i.e. $\Omega=\left\{0 \leq \xi_{1}, \xi_{2} \leq 1\right\}$ (Fig. 1). The identification of an inclusion $B_{\text {true }}$ is considered, based on four (simulated) experiments. The pressure field $u_{\text {true }}^{(m)}$ for experiment number $m$ is defined through the transmission problem

$$
\begin{aligned}
& \left.\left(\Delta+k^{2}\right) u_{\text {true }}^{(m)}=0 \quad\left(\text { in } \Omega \backslash B_{\text {true }}\right), \quad\left(\beta \Delta+\beta \gamma^{2} k^{2}\right) u_{\text {true }}^{(m)}(\boldsymbol{\xi})=0 \quad \text { (in } B_{\text {true }}\right) \\
& \left.\boldsymbol{\nabla} u_{\text {true }}^{(k)}(\boldsymbol{\xi}) \cdot \boldsymbol{n}(\boldsymbol{\xi})=g_{m} \quad(\text { on } S), \quad \llbracket u_{\text {true }} \rrbracket=\llbracket \rho^{-1} \nabla u_{\text {true }}^{(k)} \cdot \boldsymbol{n} \rrbracket=0 \quad \text { (on } \partial B_{\text {true }}\right)
\end{aligned}
$$

where $\Delta$ denotes the two-dimensional Laplacian operator, $k$ is the wavenumber, and the prescribed excitation $g_{m}$ is constant on each side $S_{q}$ of $\Omega$ (Fig. 1): $g_{m}=\delta_{m q}$ on $S_{q}$. Problem (61) is such that $S_{\mathrm{T}}=S, S_{u}=\emptyset$. All computations were performed using a Matlab implementation of the finite element method based on three-noded, piecewise-linear, triangular elements. The cost function $E$ is defined by (46), with the "Neumann" solution corresponding to problem (61) with $B_{\text {true }}=\emptyset$ and the "Dirichlet" solution corresponding to prescribing $u=u_{\mathrm{obs}}^{(m)}$ on $S$, where $u_{\mathrm{obs}}^{(m)}=u_{\text {true }}^{(m)}$ (the effect of noise not being considered in this simple example). The topological sensitivity $\mathcal{T}(\boldsymbol{z}, T)$ of $E$ is given (following an analysis similar to that of Sec. 3 where disk-shaped nucleating inclusions are considered) by
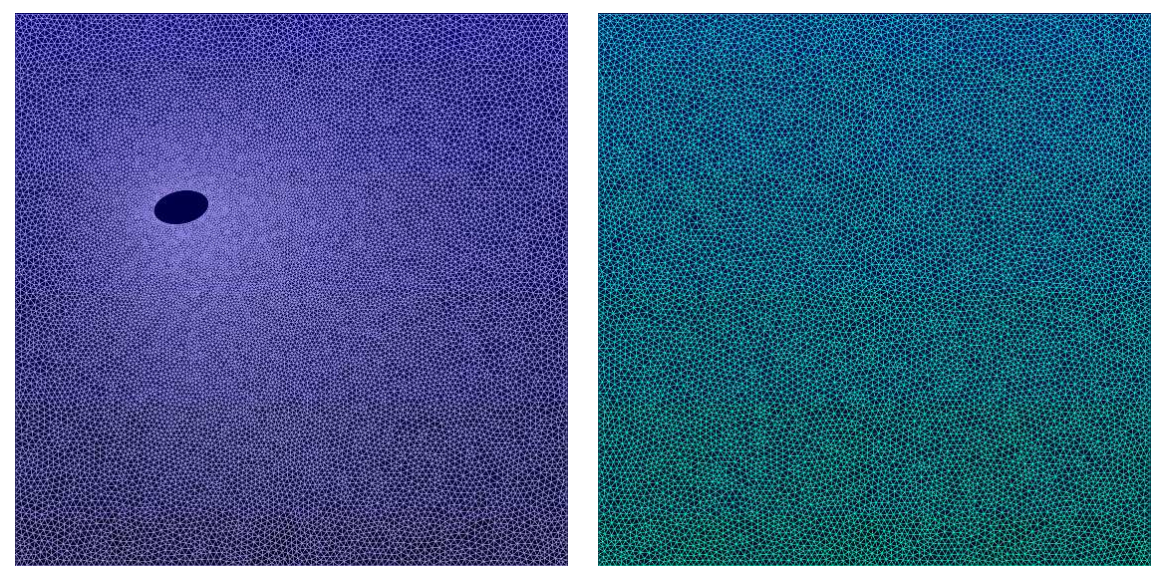

Fig. 2. Identification of a single scatterer: meshes used for generating the synthetic data (left) and computing the topological sensitivity (right). 


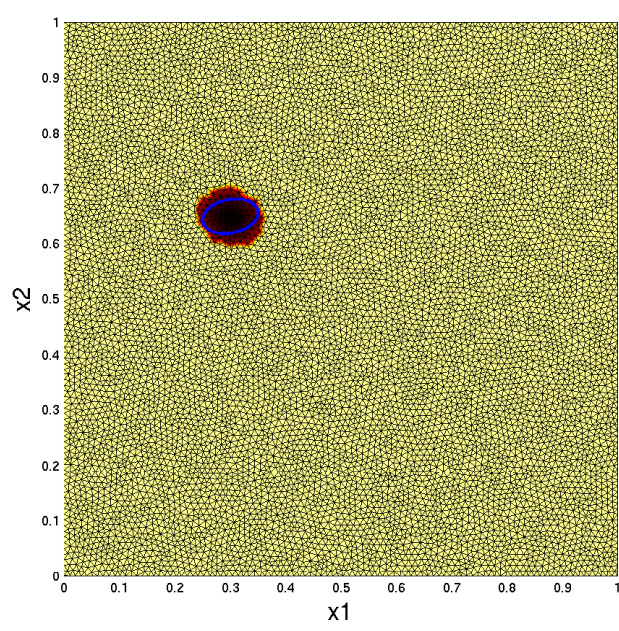

(a) $k^{2}=120$

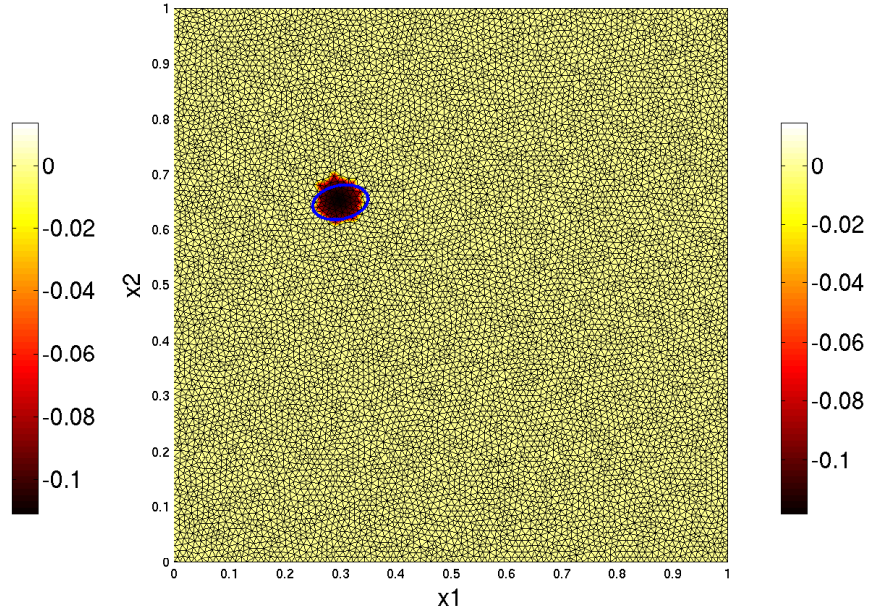

(b) $k^{2}=220$

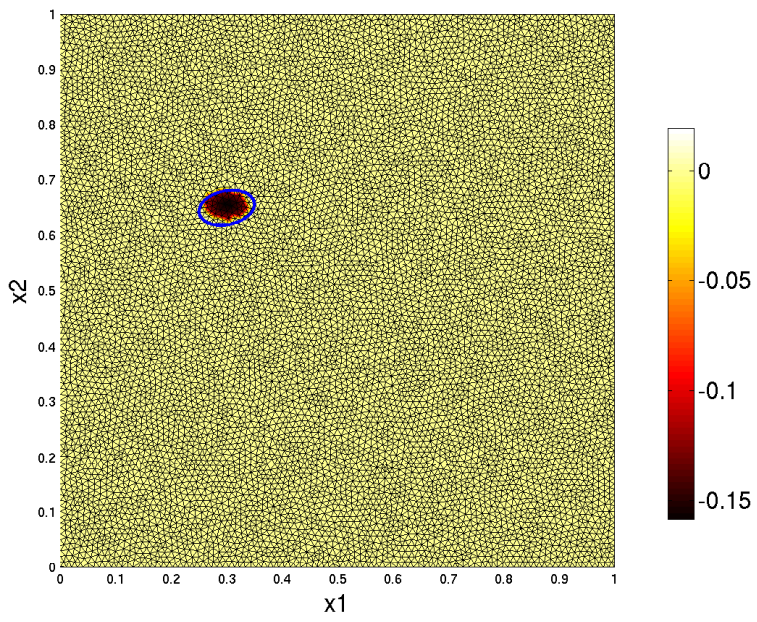

(c) $k^{2}=420$

Fig. 3. Identification of a single scatterer: distribution of $\mathcal{T}_{\alpha}$ with $\alpha=0.75$.

$$
\begin{array}{r}
\mathcal{T}_{m}(\boldsymbol{z}, T)=\frac{2(1-\beta)}{1+\beta} \operatorname{Re}\left[\boldsymbol{\nabla} w^{\mathrm{D}} \cdot \boldsymbol{A} \cdot \boldsymbol{\nabla} \bar{u}^{\mathrm{D}}+\boldsymbol{\nabla} w^{\mathrm{N}} \cdot \boldsymbol{A} \cdot \boldsymbol{\nabla} \bar{u}^{\mathrm{N}}+\frac{(1-\beta)}{1+\beta} \boldsymbol{\nabla}\left(u^{\mathrm{N}}-u^{\mathrm{D}}\right) \cdot \boldsymbol{\nabla}\left(\bar{u}^{\mathrm{N}}-\bar{u}^{\mathrm{D}}\right)\right](\boldsymbol{z}) \\
+\left(\beta \gamma^{2}-1\right) k^{2}\left(w^{\mathrm{D}} \bar{u}^{\mathrm{D}}+w^{\mathrm{N}} \bar{u}^{\mathrm{N}}\right)(\boldsymbol{z})
\end{array}
$$

On combining data from experiments $1 \leq m \leq 4$ through an additive cost functional $E=\sum_{m} E^{(m)}$, the corresponding topological sensitivity is simply $\mathcal{T}=\sum_{m} \mathcal{T}^{(m)}$, where each $\mathcal{T}^{(m)}$ is evaluated according to (62) with the free and adjoint fields defined using data $g^{(m)}$ and $u_{\text {obs }}^{(m)}$.

Like in earlier works such as $[5,7,12,16,25]$, a heuristic identification method based on the computation of the TS field $\mathcal{T}(\boldsymbol{z})$ consists in considering locations where $\mathcal{T}(\boldsymbol{z})$ attains its lowest negative values as the most likely sites for a defect, as $\mathcal{T}(\boldsymbol{z})<0$ indicates that the cost functional will decrease under the nucleation of a sufficiently small inclusion at $\boldsymbol{z}$. To focus on areas of $\Omega$ where $\mathcal{T}$ attains sufficiently low (negative) values, a thresholded version $\mathcal{T}_{\alpha}$ of $\mathcal{T}$ depending on a cut-off parameter $\alpha$ is defined (with the 
implicit assumption that $\left.\mathcal{T}^{\text {min }}<0\right)$ by

$$
\mathcal{T}_{\alpha}(\boldsymbol{z})=\left\{\begin{array}{ll}
\mathcal{T}(\boldsymbol{z}) & \left(\mathcal{T} \leq \alpha \mathcal{T}^{\text {min }}\right), \\
0 & \left(\mathcal{T}>\alpha \mathcal{T}^{\text {min }}\right)
\end{array} \quad \text { with } \mathcal{T}^{\text {min }}=\min _{\boldsymbol{z}} \mathcal{T}(\boldsymbol{z}), \alpha<1\right.
$$

Consider the identification of a single scatterer $B^{\text {true }}$ with material parameters $\beta=0.2, \gamma=0.5$ and the following geometrical parameters: major semiaxis $\sqrt{26} / 100,3 \sqrt{26} / 500$, minor semiaxis $(0.30,0.65)$ and inclination (i.e. angle between the $\xi_{1}$-direction and the major semiaxis) $\tan ^{-1}(1 / 5)$. The meshes used for generating the synthetic data $u_{\text {true }}^{(m)}$ and for computing $u^{\mathrm{D}, \mathrm{N}}, w^{\mathrm{D}, \mathrm{N}}$ and $\mathcal{T}$ (Fig. 2) feature 16268 and 9841 DOFs, respectively. For given data $u_{\text {obs }}$, all computations required for evaluating the field $\mathcal{T}(\boldsymbol{z})$ are performed on the latter mesh (for the reference domain), the former being used here only for creating the synthetic data used in numerical experiments.

Figure 3 shows the distribution of $\mathcal{T}_{\alpha}$ computed for the previously-defined exact simulated data (with $\alpha=0.75$ ), for three testing frequencies such that $k^{2}=120,220,420$. The location of the defect is correctly pinpointed for the three cases.

\section{Conclusion}

The main result of this article is the formulation of the topological sensitivity of an energy-like cost functional which is well suited for various identification problems. Importantly, the topological sensitivity field $\mathcal{T}(\boldsymbol{z})$ is expressed as a combination of four elastodynamic fields defined on the reference solid, enabling all com putations to be done on that configuration. Examination of this field permits a qualitative identification of defects in a non-iterative way, e.g. providing a good initial guess for a subsequent fullfledged, and computationally more demanding, minimization. The derivation of the topological sensitivity is here presented for a specific choice of the energy-like cost functional, but the pattern of analysis used is expected to allow establishing corresponding results for other versions of energy cost functionals, and also to formulations in the time domain.

\section{References}

[1] Ammari, H., Kang, H. Reconstruction of small inhomogeneities from boundary measurements. Lecture Notes in Mathematics 1846. Springer-Verlag (2004).

[2] Ammari, H., Kang, H. Polarization and Moment Tensors With Applications to Inverse Problems and Effective Medium Theory. Applied Mathematical Sciences, Vol. 162. Springer-Verlag (2007).

[3] Ammari, H., Khelifi, A. Electromagnetic scattering by small dielectric inhomogeneities. J. Maths Pures Appl., 82:749842 (2003).

[4] Andrieux, S., Ben Abda, A. Solving Cauchy problems by minimizing an energy-like functional. Inverse Problems, 22:115-133 (2006).

[5] Ben Abda, A., Hassine, M., Jaoua, M., Masmoudi, M. Topological sensitivity analysis for the location of small cavities in Stokes flow. SIAM J. Contr. Opt., 48:2871-2900 (2009).

[6] Bonnet, M. Boundary integral equation methods for solids and fluids. John Wiley \& Sons (1999).

[7] Bonnet, M., Guzina, B. B. Sounding of finite solid bodies by way of topological derivative. Int. J. Num. Meth. Eng., 61:2344-2373 (2004)

[8] Cakoni, F., Colton, D. Qualitative methods in inverse scattering theory. Springer-Verlag (2006).

[9] Cedio-Fengya, D. J., Moskow, S., Vogelius, M. Identification of conductivity imperfections of small diameter by boundary measurements. Continuous dependence and computational reconstruction. Inverse Problems, 14:553-595 (1998). 
[10] Chavent, G., Kunisch, K., Roberts, J. E. Primal-dual formulations for parameter estimation problems. Computational and Applied Mathematics, 18:173-229 (1999).

[11] Deraemaeker, A., Ladevèze, P., Leconte, Ph. Reduced bases for model updating in structural dynamics based on constitutive relation error. Comp. Meth. Appl. Mech. Eng., 191:2427-2444 (2002).

[12] Feijóo, G. R. A new method in inverse scattering based on the topological derivative. Inverse Problems, 20:1819-1840 (2004).

[13] Feissel, P., Allix, O. Modified constitutive relation error identification strategy for transient dynamics with corrupted data: the elastic case. Comp. Meth. Appl. Mech. Eng., 196:1968-1983 (2006).

[14] Geymonat, G., Pagano, S. Identification of mechanical properties by displacement field measurement: a variational approach. Meccanica, 38:535-545 (2003).

[15] Gockenbach, M., Khan, A. A. An Abstract Framework for Elliptic Inverse Problems: Part 2. An Augmented Lagrangian Approach. Math. Mech. Solids, 14:517 - 539 (2009).

[16] Guzina, B. B., Bonnet, M. Topological derivative for the inverse scattering of elastic waves. Quart. J. Mech. Appl. Math., 57:161-179 (2004).

[17] Guzina, B. B., Bonnet, M. Small-inclusion asymptotic of misfit functionals for inverse problems in acoustics. Inverse Problems, 22:1761-1785 (2006).

[18] Guzina, B. B., Chikichev, I. From imaging to material identification: a generalized concept of topological sensitivity. J. Mech. Phys. Solids, 55:245-279 (2007).

[19] Knowles, I. Parameter identification for elliptic problems. J. Comp. Appl. Math., 131:175-194 (2001).

[20] Kohn, R., McKenney, A. Numerical implementation of a variational method for electrical impedance tomography. Inverse Problems, 6:389-414 (1990).

[21] Kohn, R.V., Lowe, B.D. A variational method for parameter identification. Math. Mod. Num. Anal., 22:293-315 (1988).

[22] Ladevèze, P., Chouaki, A. Application of a posteriori error estimation for structural model updating. Inverse Problems, 15:49-58 (1999).

[23] Ladevèze, P., Leguillon, D. Error estimate procedure in the finite element method and applications. SIAM J. Numer. Anal., 20:485-509 (1983).

[24] Martin, P. A. Acoustic scattering by inhomogeneous obstacles. SIAM J. Appl. Math., 64:297-308 (2003).

[25] Masmoudi, M., Pommier, J., Samet, B. The topological asymptotic expansion for the Maxwell equations and some applications. Inverse Problems, 21:547-564 (2005).

[26] Maz'ya, V., Nazarov, S. A., Plamenevskii, B. A. Asymptotic theory of elliptic boundary value problems under a singular perturbation of the domains (vols. 1 and 2). Birkhaüser (2000).

[27] Mura, T. Micromechanics of Defects in Solids. Martinus Nijhoff (1982).

[28] Potthast, R. A survey on sampling and probe methods for inverse problems. Inverse Problems, 22:R1-R47 (2006).

[29] Snieder, R. General theory of elastic wave scattering. In R. Pike, P. C. Sabatier (eds.), Scattering: scattering and inverse scattering in pure and applied science, pp. 528-542. Academic Press (2002). 